\title{
Bimodal Behavior in the Zonal Mean Flow of a Baroclinic $\beta$-Channel Model
}

\author{
S. Kravtsov, A. W. Robertson, ${ }^{*}$ AND M. GHIL ${ }^{+}$ \\ Department of Atmospheric and Oceanic Sciences and Institute of Geophysics and Planetary Physics, University of California, \\ Los Angeles, Los Angeles, California
}

(Manuscript received 5 December 2003, in final form 15 October 2004)

\begin{abstract}
The dynamical origin of midlatitude zonal-jet variability is examined in a thermally forced, quasigeostrophic, two-layer channel model on a $\beta$ plane. The model's behavior is studied as a function of the bottom-friction strength.

Two distinct zonal-flow states exist at realistic, low, and intermediate values of the bottom drag; these two states are maintained by the eddies and differ mainly in terms of the meridional position of their climatological jets. The system's low-frequency evolution is characterized by irregular transitions between the two states.

For a given branch of model solutions, the leading stationary and propagating empirical orthogonal functions are related to eigenmodes of the model's dynamical operator, linearized about the climatological state on this branch. Nonlinear interactions between these modes are instrumental in determining their relative energy level. In particular, the stationary modes' self-interaction is shown to vanish. Thus, these modes do not exchange energy with the mean flow and, consequently, dominate the lowest-frequency behavior in the model. The leading stationary mode resembles the observed annular mode in the Southern Hemisphere.

The bimodality is due to nonlinear interactions between nearly equivalent barotropic, stationary, and propagating modes, while the synoptic eddies play a modest role in determining the relative persistence of the two states. The role of synoptic eddies is very substantial only at unrealistically high values of the bottom drag, where they give rise to ultralow frequency variability by modifying the jet in a way that reinforces generation of the eddy field. This type of behavior is related to the presence of a homoclinic orbit in the model's phase space and is not apparent for more realistic, lower values of the bottom drag.
\end{abstract}

\section{Introduction}

In this paper, we study the origin of the zonally symmetric component of extratropical atmospheric variability. Midlatitude atmospheric behavior is characterized by a variety of spatial and temporal scales. Major weather phenomena are associated with fast baroclinic waves, whose breaking forms synoptic eddies; the spatial structures of these baroclinic phenomena vary with height, and their time scales are on the order of a week and shorter. In contrast, the midlatitude low-frequency

\footnotetext{
* Current affiliation: International Research Institute for Climate Prediction (IRI), Palisades, New York.

+ Additional affiliation: Département Terre-AtmosphèreOcéan and Laboratoire de Météorologie Dynamique/IPSL, Paris, France.
}

Corresponding author address: Dr. Sergey Kravtsov, Dept. of Atmospheric and Oceanic Sciences and Institute of Geophysics and Planetary Physics, University of California, Los Angeles, 405 Hilgard Ave., Los Angeles, CA 90095-1565.

E-mail: sergey@atmos.ucla.edu variability (LFV), whose time scale is longer than that of synoptic eddies, is predominantly equivalent barotropic (Wallace 1983).

LFV modes with a pronounced zonally symmetric component are often referred to as annular modes (Wallace 2000). The annular mode in the Northern Hemisphere (NH) is called the Arctic Oscillation (AO; Deser 2000; Thompson and Wallace 2000; Thompson et al. 2000; Wallace 2000; Robertson 2001); it is strongly related to a more regional North Atlantic Oscillation (NAO; Hurrel 1995). In the Southern Hemisphere (SH), the so-called zonal-flow vacillation (Hartmann 1995; Hartmann and Lo 1998; Feldstein and Lee 1998; Lorenz and Hartmann 2001; Koo et al. 2003) dominates. Both modes stand out as the leading empirical orthogonal function (EOF) of either low-pass-filtered (AO) or zonally averaged (zonal-flow vacillation) data; the quantity that characterizes the time dependence of zonal-flow vacillation in the $\mathrm{SH}$ is called the zonal index (Feldstein and Lee 1998).

The annular modes consist of meridional displacements of the zonally averaged zonal jet. The next EOF of the zonal mean flow in both hemispheres is associ- 
TABLE 1. Differences between the present model and the one used in Kravtsov et al. (2003).

\begin{tabular}{lll}
\hline \hline \multicolumn{1}{c}{ Parameter } & \multicolumn{1}{c}{ Kravtsov et al. (2003) } & \multicolumn{1}{c}{ Present model } \\
\hline$k^{-1}$ & 15.4 days & Variable \\
$\beta$ & $1.87 \times 10^{-11} \mathrm{~m}^{-1} \mathrm{~s}^{-1}$ & $2 \times 10^{-11} \mathrm{~m}^{-1} \mathrm{~s}^{-1}$ \\
$A_{H}$ & $-2 \times 10^{16} \mathrm{~m}^{4} \mathrm{~s}^{-1}$ & $-1.5 \times 10^{16} \mathrm{~m}^{4} \mathrm{~s}^{-1}$ \\
Planetary mode-0 damping & Yes & No \\
Lateral boundary conditions & Free slip & No slip \\
Wind speed dependence in $H_{\mathrm{SL}}$ & No & Yes \\
Insolation & $R=182.04-162.92 \sin \left(2 y / a_{E}\right)$ & $R=190-165 \sin \left(2 y / a_{E}\right)$ \\
Sea surface temperature & $T_{s}=12.4-17.0 \sin \left(2 y / a_{E}\right)$ & $T_{s}=13-17 \sin \left(2 y / a_{E}\right)$ \\
\hline
\end{tabular}

ated with irregular weakening and strengthening of the jet (Lorenz and Hartmann 2001, 2003). These modes have been also obtained in idealized numerical models (Robinson 1991, 1996, 2000; Yu and Hartmann 1993; Feldstein and Lee 1996; Lee and Feldstein 1996; Koo and Ghil 2002; Kravtsov et al. 2003).

The mechanisms that govern this behavior are not fully understood: observational and theoretical results give rise to controversial interpretations. One unresolved issue concerns the manner in which synoptic eddies interact with the annular modes. These modes may be selected by the so-called synoptic eddy feedback that involves anomalous generation of synoptic eddies in a way that reinforces the zonal wind anomaly (Namias 1953; Shutts 1983; Illari 1984; Robertson and Metz 1989, 1990; Robinson 1991; Branstator 1992, 1995; Yu and Hartmann 1993; Cai and Van den Dool 1994; Feldstein and Lee 1998; Lorenz and Hartmann 2001, 2003 ). On the other hand, Feldstein and Lee (1996) and Lee and Feldstein (1996) suggest that eddy feedback is not important for the evolution of the zonal index. Robinson $(1996,2000)$ has argued that the eddy feedback is significant only if the bottom drag is sufficiently strong. Kravtsov et al. (2003) present results consistent with this hypothesis: in their two-layer baroclinic channel model with a relatively low bottom drag, synoptic eddies are modulated by LFV, but are fairly passive dynamically, while the LFV itself is due to weakly interacting barotropic modes.

Another puzzling property of extratropical LFV is its strong association with eigenmodes of the system linearized about the observed climatological state (Branstator 1992; Metz 1994; Da Costa and Vautard 1997; Itoh and Kimoto 1999; Kravtsov et al. 2003; Watanabe and Jin 2003). This association has prompted the formulation of linear stochastic models of zonal-flow vacillation (Kidson and Watterson 1999; Feldstein 2000). In contrast, S. Koo and colleagues (Koo and Ghil 2002; Koo et al. 2003) recently presented a nonlinear framework for zonal-flow vacillation, based on the paradigm of multiple flow regimes (Reinhold and Pierrehumbert 1982; Legras and Ghil 1985; Marshall and Molteni 1993). The flow regimes in this context are associated with two persistent zonal-jet states; zonal-flow vacillation is the result of irregular transitions between them due to wave-mean flow interactions.
In this paper, we attempt to reconcile linear and nonlinear theories of extratropical LFV. To this end, we study in greater depth a model that is very similar to that of Kravtsov et al. (2003). Following Robinson (1996, 2000) and Koo and Ghil (2002), we study this model's sensitivity to variations of bottom drag and find that the role of the synoptic eddy feedback depends strongly on this parameter.

The model is formulated in section 2 and appendix A, and its zonally averaged and three-dimensional climates are described in sections 3 and 4, respectively; multiple regimes of zonal mean flow characterize model behavior at the more realistic, moderate and low values of bottom drag only. In section 5, we discuss linear and nonlinear aspects of the system's variability and relate them to each other. A summary and discussion of the results follow in section 6. Appendix B contains a discussion of the first few bifurcations that lead to the multiple regime behavior described in sections 3-5; they occur at high bottom drag, the only parameter range where synoptic eddy feedback is important.

\section{Model formulation and methodology}

The present model is a slight variation of the one studied by Kravtsov and Robertson (2002) and Kravtsov et al. (2003). The model version used here is summarized below and the differences between this version and the previous one are listed in Table 1.

\section{a. Model formulation}

The model geometry is depicted in Fig. 1, with a zonal cross section through the model in the top panel and a plan view at the bottom. To model the effects of land-sea contrast on the atmospheric circulation, an oceanic region is included in which the sea surface temperature is prescribed. This oceanic region represents a midlatitude portion of the North Atlantic Basin, which is approximately $60^{\circ}$ wide at $45^{\circ} \mathrm{N}$, and extends from $16^{\circ}$ to $66^{\circ} \mathrm{N}$. The insulating land strip just to the north of the ocean basin mimics the presence of polar sea ice and introduces meridional asymmetry in the lower atmospheric boundary conditions, which turns out to be important for the model's climatology and its LFV. Atmospheric latitudinal boundaries are situated at $16^{\circ}$ and 


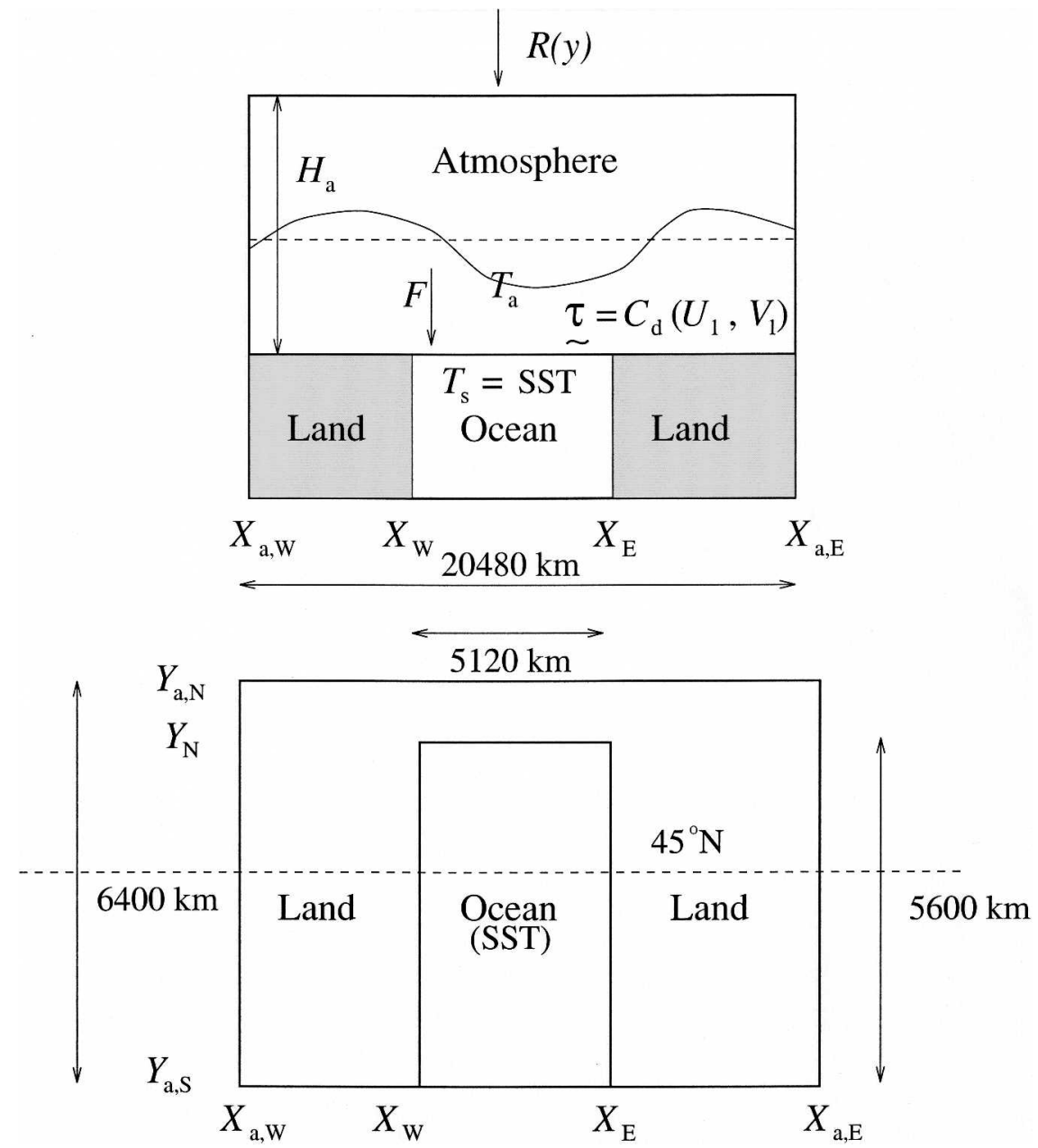

FIG. 1. Schematic diagram of model geometry. (top) Vertical cross section; (bottom) plan view. Land areas are shaded. The ocean region extends longitudinally from $X_{W}=3520 \mathrm{~km}$ to $X_{E}=8640 \mathrm{~km}$, and latitudinally from $Y_{S}=-3200 \mathrm{~km}$ to $Y_{N}=2400 \mathrm{~km}$, with $y=0$ corresponding to $45^{\circ} \mathrm{N}$. The length of the atmospheric channel is $20480 \mathrm{~km}$, and its boundaries are located at $Y_{a, S}=Y_{S}$ and $Y_{a, N}=3200 \mathrm{~km}$ in latitude.

$74^{\circ} \mathrm{N}$ respectively. Periodic boundary conditions are assumed in the zonal direction. The atmospheric height is $H_{\mathrm{a}}=10000 \mathrm{~m}$.

We use the classical two-layer quasigeostrophic (QG) model (Pedlosky 1987) to represent midlatitude atmospheric dynamics. Such models have been used previously, with different types of lower boundary conditions (Marshall and Molteni 1993; Corti et al. 1997; Weisheimer et al. 2003) and have shown success in modeling certain aspects of midlatitude climatology and LFV. Our study differs from the previous ones by the lower and upper boundary forcing, as well as by its focus on zonal mean flow bimodality.

The governing equations for the barotropic component $\psi$ and baroclinic component $\tau$ of the streamfunction are given in appendix A. Equations (A1) and (A2), subject to no-slip conditions on the northern and southern boundaries, as well as to mass and momentum con- straints (McWilliams 1977), are discretized on a $128 \times$ 41 grid with a resolution of $160 \mathrm{~km}$ in both $x$ and $y$. They are numerically integrated using centered differences in space and leapfrog time stepping, with $\Delta t=10$ $\min$.

\section{b. Methodology}

We study the sensitivity of our model to variations of the barotropic spindown time scale $k^{-1}$, where $k$ is the bottom drag coefficient [see Eq. (A1)]. The values of $k^{-1}$ that we use range over almost two full orders of magnitude, from 0.39 to 15.4 days. A major difference between the present model and the one of Kravtsov et al. (2003) is the use of no-slip conditions along the channel's northern and southern boundaries (see Table 1). Using free-slip conditions in a wide range of bottomdrag values results in an unrealistic behavior, charac- 
terized by very strong stationary waves that arise because of the interference of waves reflected from the channel walls. Employing no-slip conditions inhibits wave reflection and results in reasonable behavior over the whole range of $k^{-1}$ we have explored.

The methodology of following changes in model behavior as a control parameter varies is rooted in dynamical systems theory, which has found an important area of applications in the atmospheric sciences (Lorenz 1963; Charney and DeVore 1979; Ghil and Childress 1987). Typically, as the control parameter changes, initially simple model solutions (e.g., steady states) undergo bifurcations that result in a more complex behavior (multiple steady states, limit cycles, period doubling bifurcations, etc.). The practical value of the method lies in its ability to identify dynamical modes that exhibit significant variations in the vicinity of such bifurcation points; these modes are the ones responsible for changes in the structure of model solutions.

We cover, therefore, a wide range of the bottom drag $k$, from unrealistically high values to more realistic intermediate and low values. This will enable us to address the dynamics of the model behavior in a regime that is most relevant to the real atmosphere. Lorenz and Hartmann $(2001,2003)$ estimate the spindown time scale $k^{-1}$ to be $k^{-1} \approx 7$ days for the $\mathrm{NH}$ and $k^{-1} \approx 9$ days for the $\mathrm{SH}$, so the realistic range of this parameter is 6 days $<k^{-1}<10$ days.

For each $k$, the model is spun up for $N_{s}=365$ days, and the control integration is run subsequently for $N=$ 3000 days. We use standard principal component (PC) analysis (Preisendorfer 1988) applied to model time series that are sampled daily to compute the dominant spatial patterns of model behavior as its leading EOFs. We identify robust modes of the system's variability that exist throughout the range of $k$ and follow the changes in these modes as the control parameter changes (sections 3 and 4). The vorticity budget is then analyzed to identify the role of these modes in the system's dynamics (section 5).

\section{Zonally averaged climate}

In spite of the land-sea contrast at the model's lower boundary, its climate possesses a pronounced zonal symmetry throughout most of the control parameter range. We therefore consider first the zonally symmetric aspects of the model's behavior and track the development of bimodality exhibited by the system in a realistic range of the bottom-drag parameter (see section 2b).

\section{a. Definitions}

A typical configuration of the zonally averaged model climate is depicted in Fig. 2 for $k^{-1}=6.7$ days.
Figure 2a shows the climatology and leading EOFs of the barotropic zonal velocity. The climate mean (heavy solid line) is characterized by a narrow climatological jet centered just to the north of the channel's axis. This north-south asymmetry in the model climate is due to the model's lower boundary forcing (see section 2a). The jet-axis position is marked by the vertical heavy solid line. We define the width of the jet $\lambda_{\text {jet }}$ as the meridional extent of the region in which the climatological jet velocity exceeds half of the maximum jet velocity $U_{\text {max }}$.

The leading EOF of the barotropic zonal velocity (light solid curve) accounts for $64 \%$ of total variance and is associated with the meridional shifts of the jet, while the next EOF (light dashed) accounts for $24 \%$ of the variance and describes the changes in the jet intensity. These two EOFs are well separated from others in terms of the variance. The distances between the jet axis and the two extrema of the leading EOF, $\Delta \lambda_{\text {jet }}^{u}$ and $\Delta \lambda_{\text {jet }}^{1}$ characterize meridional excursions of the jet.

The climatological profile of the zonal mean atmospheric temperature is shown in Fig. 2b. The jet region between $\lambda_{1}$ and $\lambda_{2}$ corresponds to an increased meridional temperature gradient.

\section{b. Multiple regimes}

The dependence of the model's climatological characteristics on the barotropic spindown time scale $k^{-1}$ is shown in Fig. 3. In Fig. 3a, the solid and dashed lines and markers indicate the jet position, dotted lines show the $\lambda_{1}$ and $\lambda_{2}$ dependencies, while $\Delta \lambda_{\text {jet }}^{u}$ and $\Delta \lambda_{\text {jet }}^{1}$ are plotted as the upper and lower error bars, respectively. The dependence of $U_{\max }$ on $k$ is plotted in Fig. 3b, with error bars showing the standard deviation of this quantity. Figure $3 \mathrm{c}$ tracks the joint distribution of jet position and intensity.

At $k^{-1} \leq 0.39$ day, the model has a single stable equilibrium; for $k^{-1}=0.39$ day, this equilibrium is marked by the large bullet in both panels. This state is characterized by an intense basinwide jet that is skewed toward the northern boundary of the channel; the skewed profile is due to the presence of the insulating land strip north of the ocean basin (see Fig. 1), which forces large atmospheric temperature gradients in this region. A similar state exists as an unstable equilibrium for all $k$ values we have explored (light solid line punctuated by small closed circles). This branch of unstable equilibria has been obtained by a quasi-Newton method, and appears to capture the only true steady states of the model. Being unstable, this branch plays no role in model behavior at realistic values of bottom drag; its role at high values of this parameter is described in appendix B.

As $k^{-1}$ increases, time-dependent behavior with an increasing degree of complexity sets in. In particular, the model climate exhibits aperiodic variations, with 


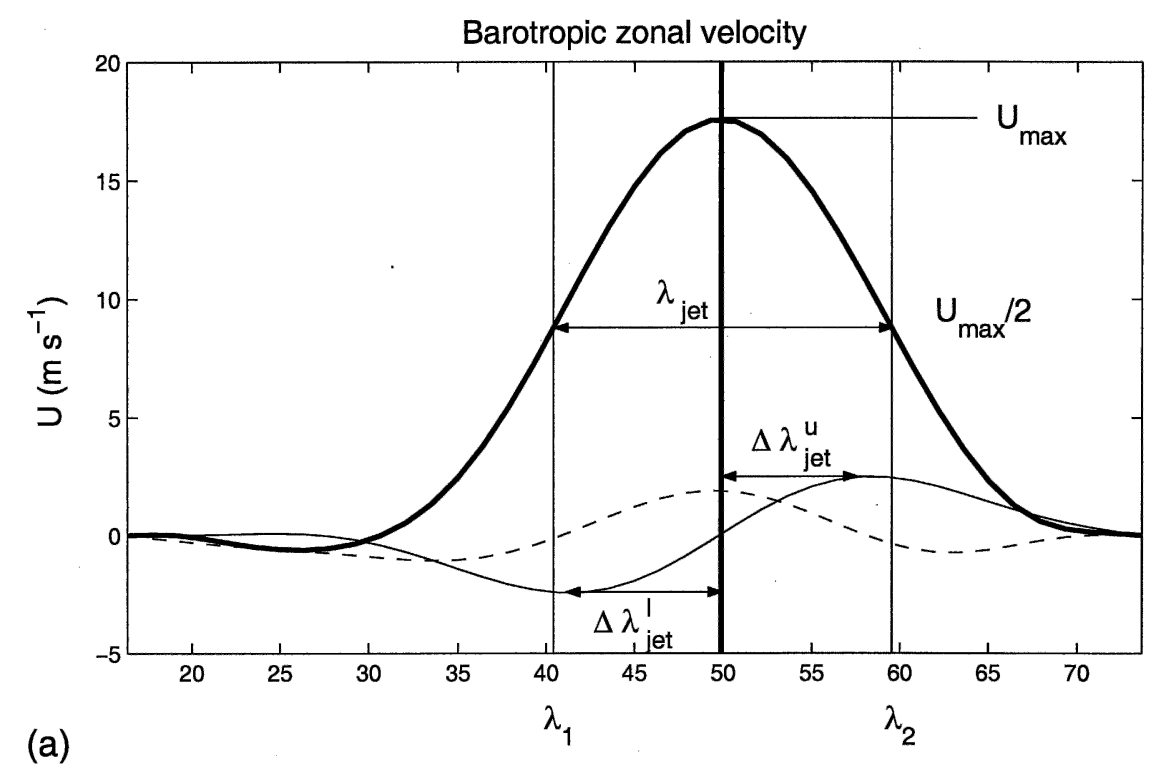

\section{(a)}

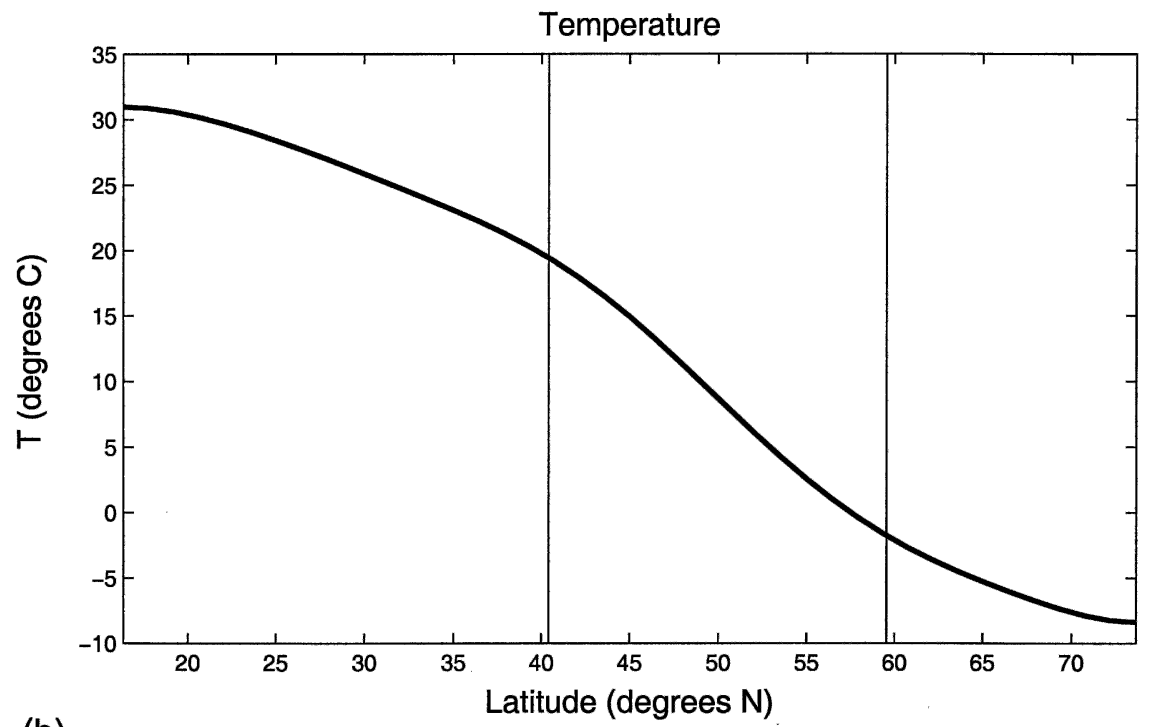

(b)

FIG. 2. Model climate in high-latitude state at $k^{-1}=6.67$ days. (top) Zonally averaged barotropic zonal velocity $\left(\mathrm{m} \mathrm{s}^{-1}\right)$ : climatology (heavy solid curve), dimensional EOF 1 (64\%) and EOF 2 (24\%) (light solid and dashed curves), and position of the jet axis (heavy solid vertical line). (bottom) Zonally averaged temperature $\left({ }^{\circ} \mathrm{C}\right)$. The distance between the light solid vertical lines at $\lambda_{1}$ and $\lambda_{2}$ in both panels defines the width of the jet.

common general characteristics for $k^{-1}>0.93$ day: the climatological jet migrates closer toward the channel's axis and becomes much narrower than at very low values of $k^{-1}<0.93$ day; the leading modes of zonally averaged variability resemble those depicted in Fig. 2a. The transition to this chaotic behavior will be described in greater detail in appendix B, while now we concentrate on the model behavior in a range of $k^{-1}$ characteristic of real atmospheric conditions.

A striking feature of the model is the presence of two distinct regimes in the zonal mean flow, namely the high-latitude and the low-latitude state. This bimodality appears for $k^{-1}>5.9$ days, and it is most pronounced for low values of the bottom drag that correspond to $k^{-1}>10$ days; at these parameter values, the system remains in one or the other flow regime, without transitions between them, and the selection of a particular regime depends on the initial flow pattern used in each integration. As the bottom drag increases, the system undergoes irregular transitions between the two regimes, with the high-latitude regime being preferred.

To track the low-latitude branch of the model's cli- 
(a)

Position of the zonal-mean jet

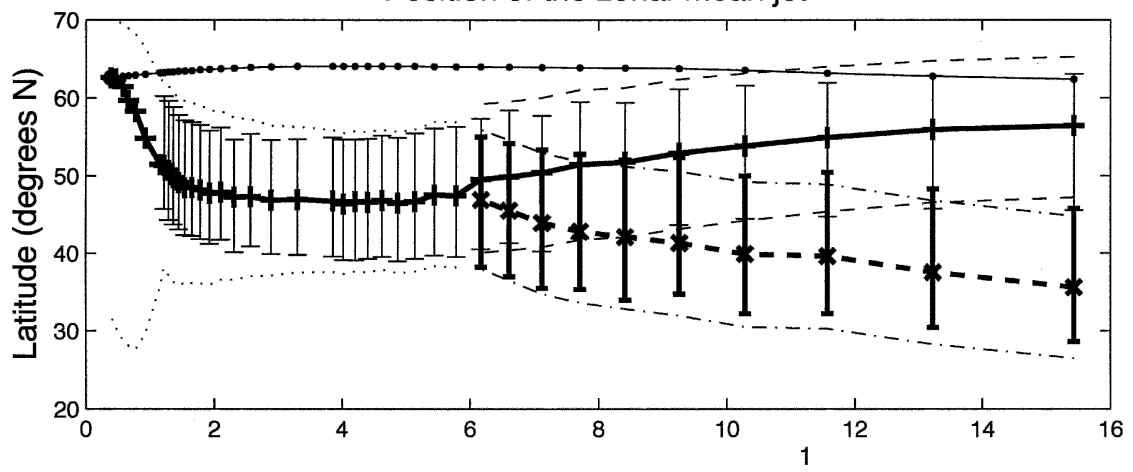

(b)

Strength of the zonal-mean jet

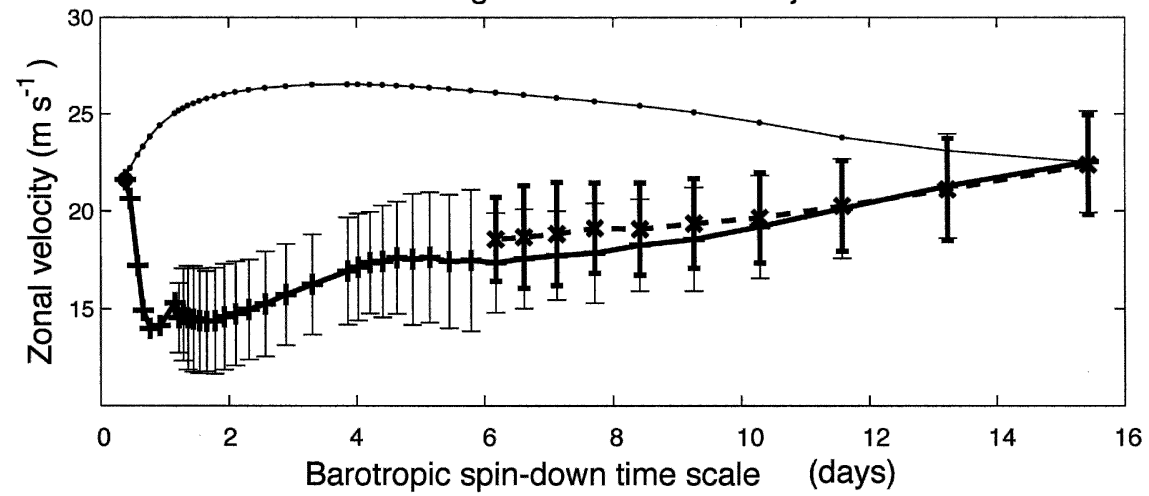

FIG. 3. Model climate as a function of the barotropic spindown time scale $k^{-1}$ (days). (a) Jet-axis position as defined in Fig. $2 ;+$ and heavy solid curve indicate the high-latitude state, while $\mathrm{x}$ and heavy dashed curve stand for the low-latitude state; $\bullet$ and light solid curve locate the unstable steady state. The width of the jet is marked by light dashed lines for the highlatitude state, by light dash-dotted lines for the low-latitude one, and by dotted lines in the unimodal region; upper and lower error bars correspond to the distances $\Delta \lambda_{\text {jet }}^{u}$ and $\Delta \lambda_{\text {jet }}^{1}$ between the jet axis and the major maximum and minimum of the leading EOF (see Fig. 2), respectively: light error bars for the high-latitude state and heavy ones for the low-latitude state. (b) Zonal-jet intensity; same symbols as in (a), except the error bars denote the standard deviation of the maximum zonally averaged barotropic zonal velocity. Large bullet marks the steady state at $k^{-1}=0.39$ day, the last value at which this state is stable.

mate, we have added a small correction term to the barotropic vorticity Eq. (A1a), of the form $k^{(0)} \nabla^{2}\left(\bar{\psi}_{k_{1}}^{(0)}\right.$ - $\left.\psi_{k_{2}}^{(0)}\right)$, where $\bar{\psi}_{k_{1}}$ is the climatological barotropic streamfunction for $k=k_{1}$, and $\psi_{k_{2}}$ is the instantaneous barotropic streamfunction for the integration with $k=$ $k_{2}>k_{1}$. This term is only present for the zonally symmetric components of the barotropic streamfunction, hence the superscript $(0) ; k^{(0)}=20$ days. This small correction is enough to keep the model in the vicinity of the low-latitude state without significantly affecting its variability about this state. The same procedure is applied to track the high-latitude state for $k^{-1}<10$ days, where the two states are situated close to each other.

For each $k$ value at which the bimodality is present, the high-latitude and low-latitude states have been defined as the long-term time means from the integrations described above. Aside from the position of the jet, these two states have similar spatial structures and exhibit comparable variability. In the following, when analyzing the variability in the bimodal regime, we will describe the model variability around a given branch of either high-latitude or low-latitude solutions, unless noted otherwise.

\section{c. Variability}

The zonally averaged variability is well described by the two leading EOFs of the barotropic zonal velocity, as shown in Fig. 2a. In Fig. 4, we plot the dimensional variance of the corresponding PCs as a function of $k$. In Figs. $4 \mathrm{a}$ and $4 \mathrm{~b}$, the variances of $\mathrm{PC} 1$ and PC 2 are shown separately for the high-latitude and low-latitude states. The error bars indicate 95\% confidence limits for a given eigenvalue (North et al. 1982; Vautard et al. 1992), based on the estimates of standard error of a covariance between Gaussian random variables. The EOF analysis above was performed around the climatological state of a given branch, with transitions 
(a) Variance of PC-1

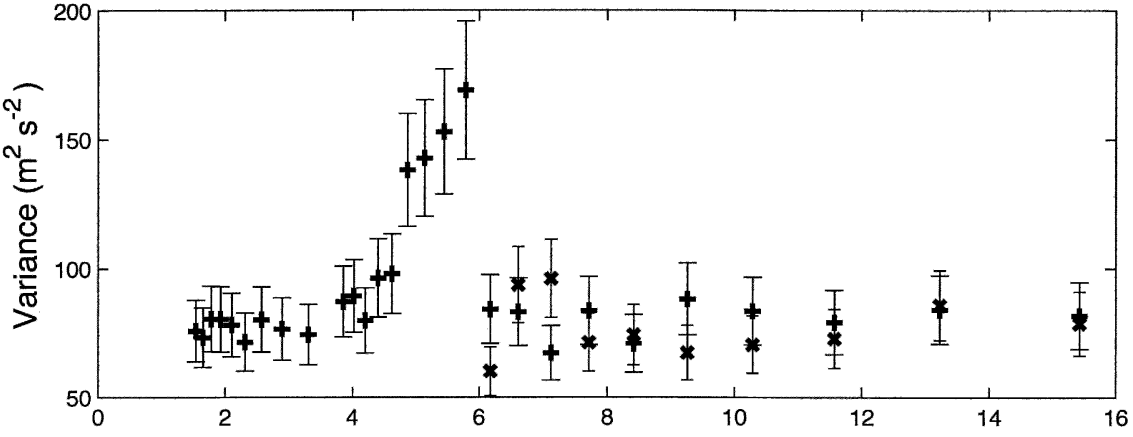

(b)

Variance of PC-2

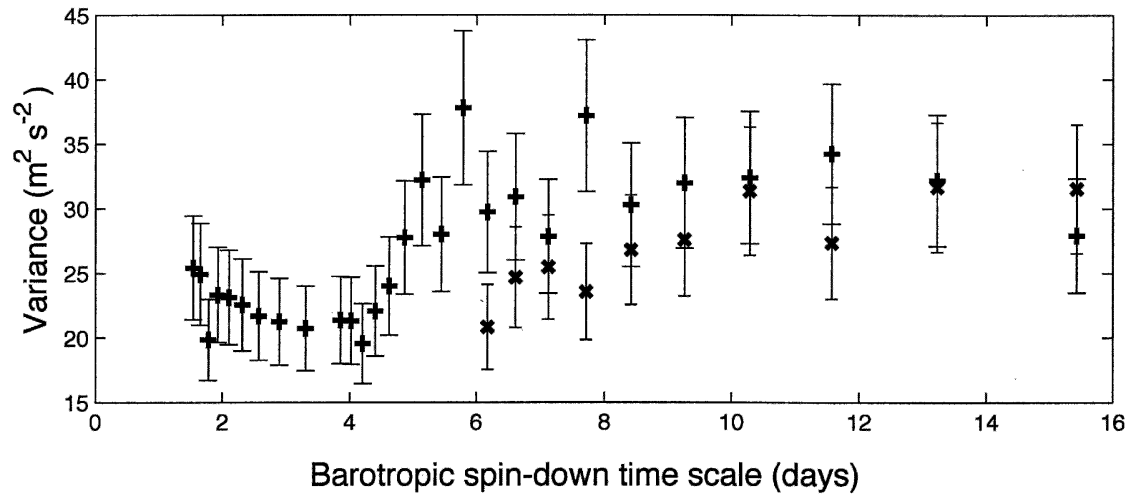

FIG. 4. Variance of the two leading PCs $\left(\mathrm{m}^{2} \mathrm{~s}^{-2}\right)$ of the zonally averaged barotropic zonal velocity (see Fig. 2a) as a function of $k^{-1}$ (days): (a) PC 1 and (b) PC 2. Error bars represent the $68 \%$ confidence interval for each eigenvalue; + for the high-latitude state and $\times$ for the low-latitude state.

between the two states being suppressed (see section 3b).

The variances of the leading PCs in the two states have comparable magnitudes. Upon passing the inferred bifurcation point $k^{-1}=5.9$ days, there is a sharp increase in PC 1 variance, accompanied by a more modest growth in PC 2 variance. After this growth, the variance of both PC 1 and PC 2 gradually decreases.

We now examine in greater detail the temporal structure of the original model (A1) solutions in a free integration without "tracking" terms, when transitions are allowed. To do so, we plot time series of the instantaneous jet-axis position for increasing bottom drag in Figs. 5a-e. The horizontal heavy solid lines show the locations of the high-latitude and low-latitude states obtained by our tracking procedure. For large $k^{-1}$ (Figs. $5 \mathrm{a}, \mathrm{b})$, the presence of the two states is immediately obvious; however, the high-latitude state is preferred over the low-latitude state. As $k^{-1}$ decreases (Figs. 5c, d), the low-latitude state is visited more frequently, but becomes less persistent. The preferred high-latitude state remains more clearly detectable; still, its persistence also decreases. For $k^{-1}=5.9$ days (Fig. 5e), no bimodality is noticeable any longer, while the jet exhibits latitudinal variations that have a much larger ampli- tude than those about either equilibrium (Fig. 5a). These results are fully consistent with the results of Fig. $4 \mathrm{a}$, since PC 1 reflects the jet's meridional shifts.

\section{Three-dimensional climate}

The multiple equilibria of the zonal flow found in section 3 are maintained by the action of longitudedependent waves. To examine the mechanisms that govern model behavior, we perform therefore fully three-dimensional diagnostics. We compute the leading modes of the system's variability for a given branch of high-latitude and low-latitude solutions (see section 3b) and follow their modifications as the control parameter changes. Doing so allows us to identify the modes that undergo significant alterations passed the inferred bifurcation point (see section $2 \mathrm{~b}$ ) and thus to infer the dynamics of the model's bimodal behavior.

\section{a. Climatology}

The model's typical climatology is shown in Fig. 6 for the high-latitude state, at $k^{-1}=6.7$ days. In Fig. $6 a$, we plot the barotropic zonal velocity (contours); and the barotropic turbulent kinetic energy 

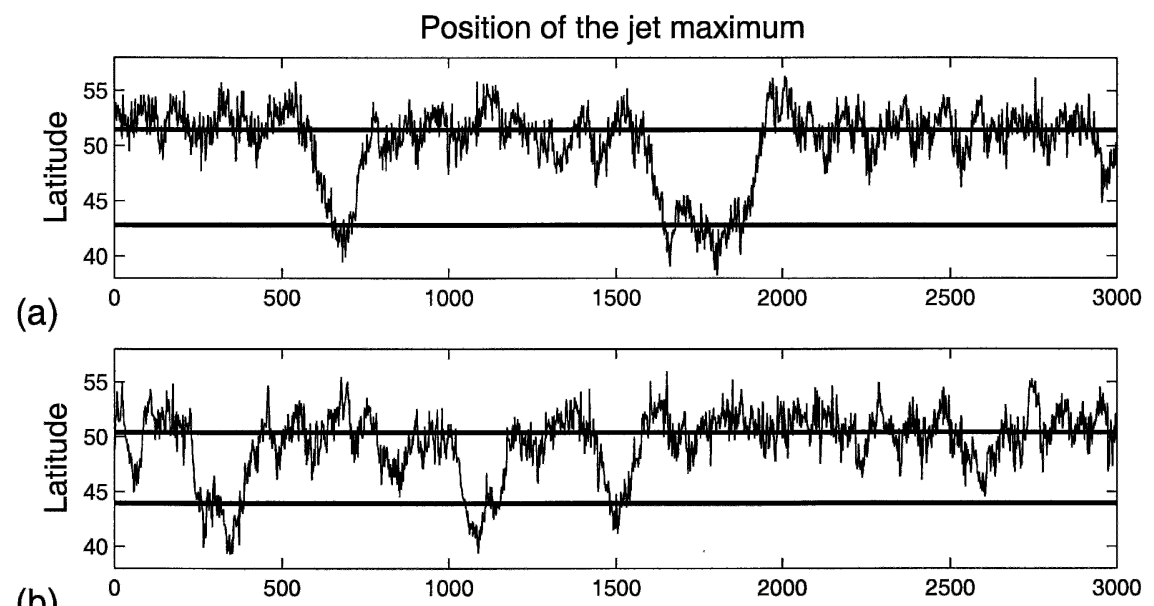

(b)
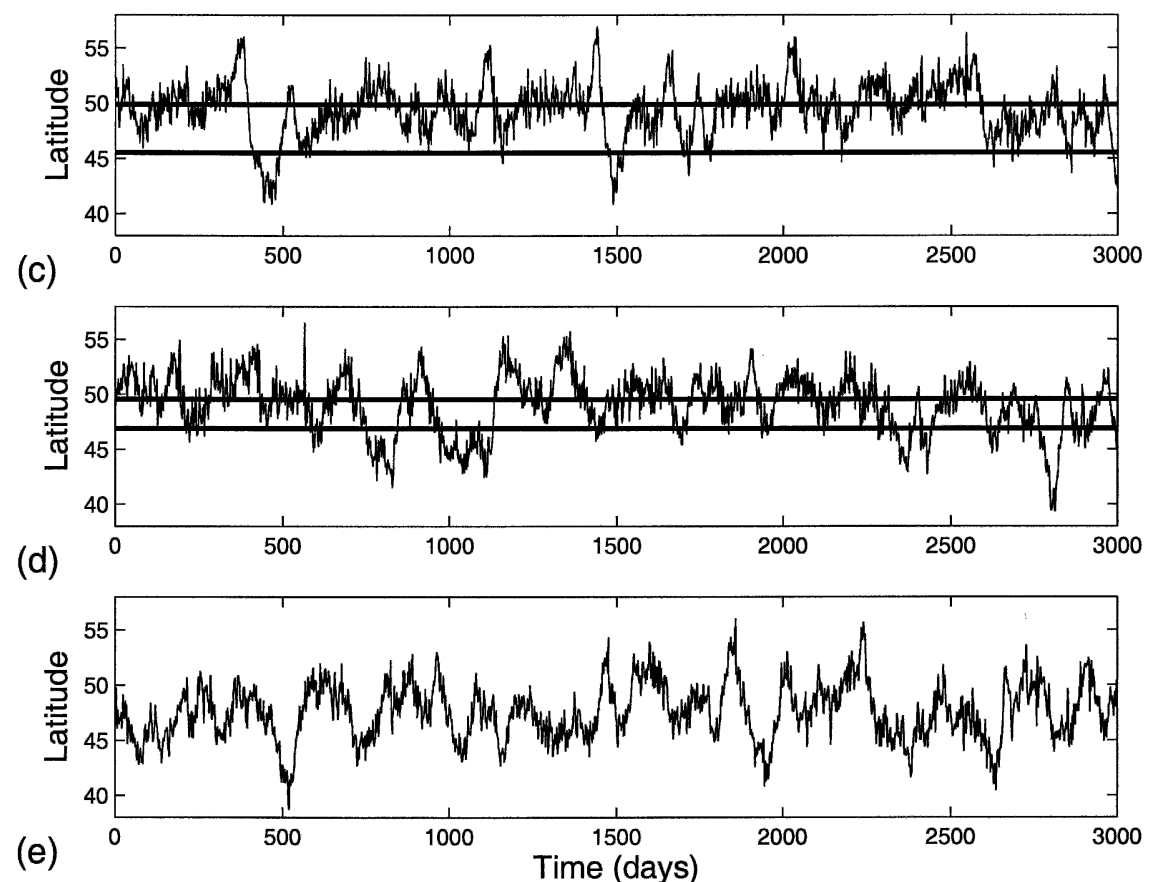

FIG. 5. Time series of the jet-center position: (a) $k^{-1}=7.7$ days; (b) $k^{-1}=7.1$ days; (c) $k^{-1}$ $=6.7$ days; (d) $k^{-1}=6.3$ days; and (e) $k^{-1}=5.9$ days. Heavy solid lines mark the climatological location of the jet in the high-latitude and low-latitude states.

$E \equiv(1 / 2)\left[\left(\partial \psi^{\prime} / \partial x\right)^{2}+\left(\partial \psi^{\prime} / \partial y\right)^{2}\right]$, where the prime denotes deviation from long-term climatology (grayscale). Climatological air temperature (contours) and temperature variance (grayscale) are plotted in Fig. 6 b.

The jet maximum is located over land, slightly to the west of the ocean basin's western shore (see Fig. 6a), while the model's storm track, seen in the variance of the temperature field, is located downstream of the jet maximum in the region of enhanced meridional temperature gradient over land (see Fig. 6b); the maximum of the equivalent barotropic LFV variability occurs at the exit of the storm track (Fig. 6a). Thus, the positions of the jet maximum, storm track, and LFV maximum are quite realistic when considered in relation to each other, but slightly farther west than in observations.

\section{b. Principal component analysis}

We performed a combined principal component (PC) analysis of the barotropic and baroclinic streamfunction fields, with no prior filtering. Typical leading stationary modes for the high-latitude state are shown in Fig. 7. The modes appear in the $\mathrm{PC}$ analysis as EOF 3 [column (a)] and EOF 8 [column (b)], and we refer to these EOFs as mode 1 and mode 2, respectively.

Both modes have a pronounced zonally symmetric 


\section{Barotropic $\mathrm{U}$ (contours; $\mathrm{m} \mathrm{s}^{-1}$ ) and TKE (shading; $\mathrm{m}^{2} \mathrm{~s}^{-2}$ )}

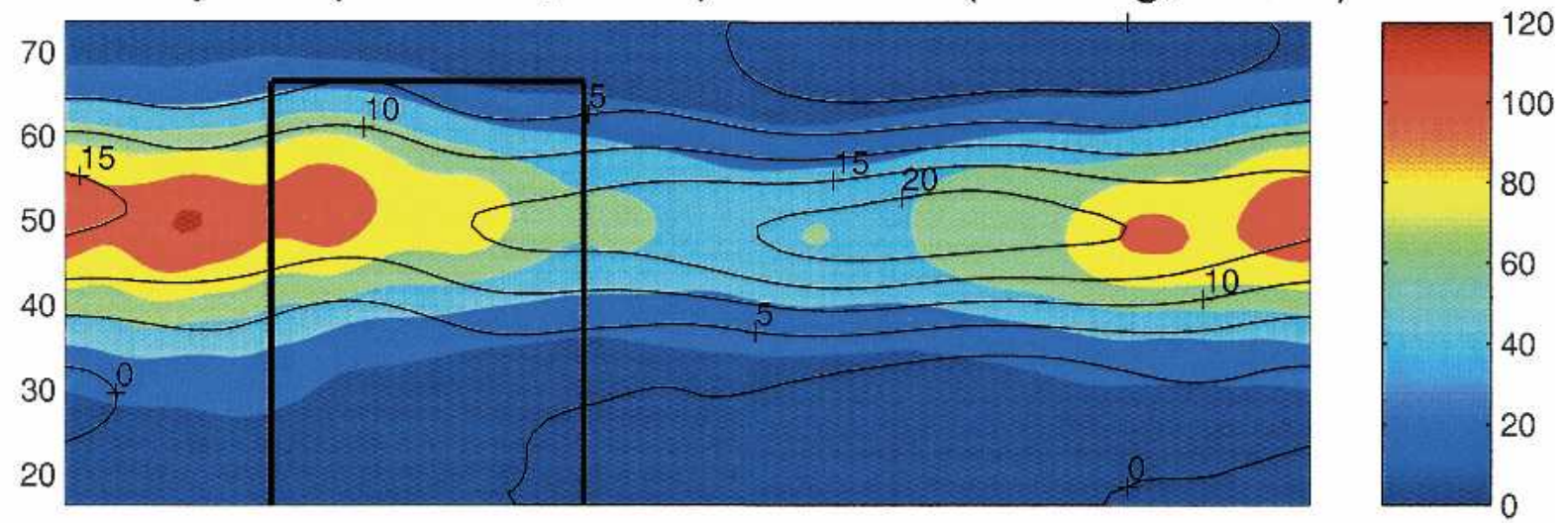

(a)

\section{$\mathrm{T}_{\mathrm{a}}$ (contours; ${ }^{\circ} \mathrm{C}$ ) and $\mathrm{T}_{\mathrm{a}}$ STD (shading; ${ }^{\circ} \mathrm{C}^{2}$ )}

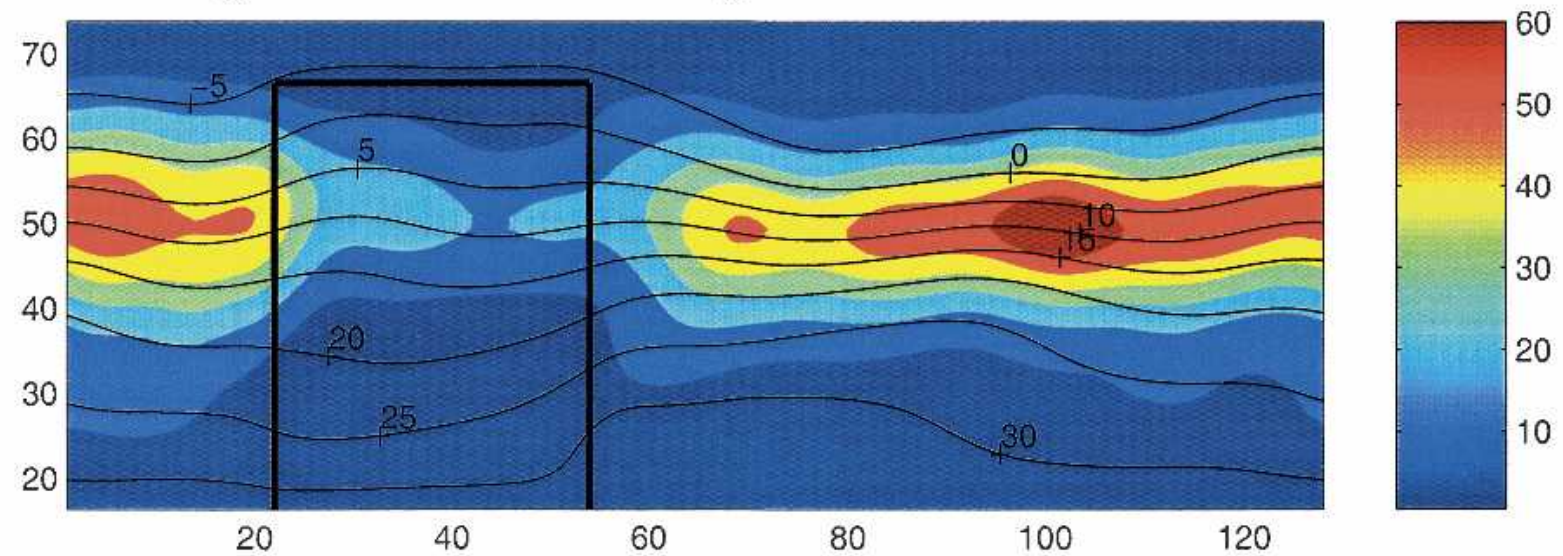

(b)

FIG. 6. Model climatology in the high-latitude state; $k^{-1}=6.7$ days. (a) Barotropic zonal velocity (contours; $\mathrm{m} \mathrm{s}^{-1}$ ) and turbulent kinetic energy (shading; $\mathrm{m}^{2} \mathrm{~s}^{-2}$ ); and (b) atmospheric temperature (contours; ${ }^{\circ} \mathrm{C}$ ) and temperature variance (shading; ${ }^{\circ} \mathrm{C}^{2}$ ). Sidebars show color scale; contour intervals (CI) are 5 units in each panel. Heavy solid lines mark the ocean's coastline.

component. They account for only a relatively small fraction of total variance-10\% for mode 1 and $3 \%$ for mode 2-but are among the three dominant modes in terms of the variance contained at intraseasonal and longer time scales (not shown) and dominate the stationary variance at all frequencies (see also Vautard et al. 1988). For these reasons, the two modes are related to the leading EOFs of the zonally averaged fields (see section 3). Performing zonal averaging of these EOFs' streamfunction and taking the $y$ derivative of the resulting fields to get zonal velocity reproduces the structure of the zonally averaged EOFs in Fig. 2a.

Mode 1 consists of meridional shifts of the jet that are slightly modulated in longitude; the temporal correlation of this mode with its zonally averaged counterpart is 0.87 . Mode 2 describes changes in the jet intensity in the presence of some zonal modulation; it corresponds to EOF 2 of the zonally averaged fields, with the tem- poral correlation between the two equal to 0.81 . Both modes are predominantly equivalent barotropic, with the upper- and lower-layer streamfunction (upper and lower panels in each column, respectively) having spatial patterns that are nearly in phase, but have a larger magnitude in the upper layer.

In addition to these stationary modes, the system has a number of propagating wave modes: the EOF 1-EOF 2 pair shares a wave- 4 pattern, while EOFs 4 and 5, 6 and 7,9 and 10 correspond to waves 5, 3, and 6, respectively. The two members of each pair have comparable variances, as well as the same spatial and temporal characteristics: they have the same temporal period and are in quadrature with each other, in both time and space. The spatial pattern of the first member of each pair is shown in Fig. 8.

Waves 4 and 3 (Figs. 8a,c) account for about 50\% and $8 \%$ of total variance, respectively, and are nearly 

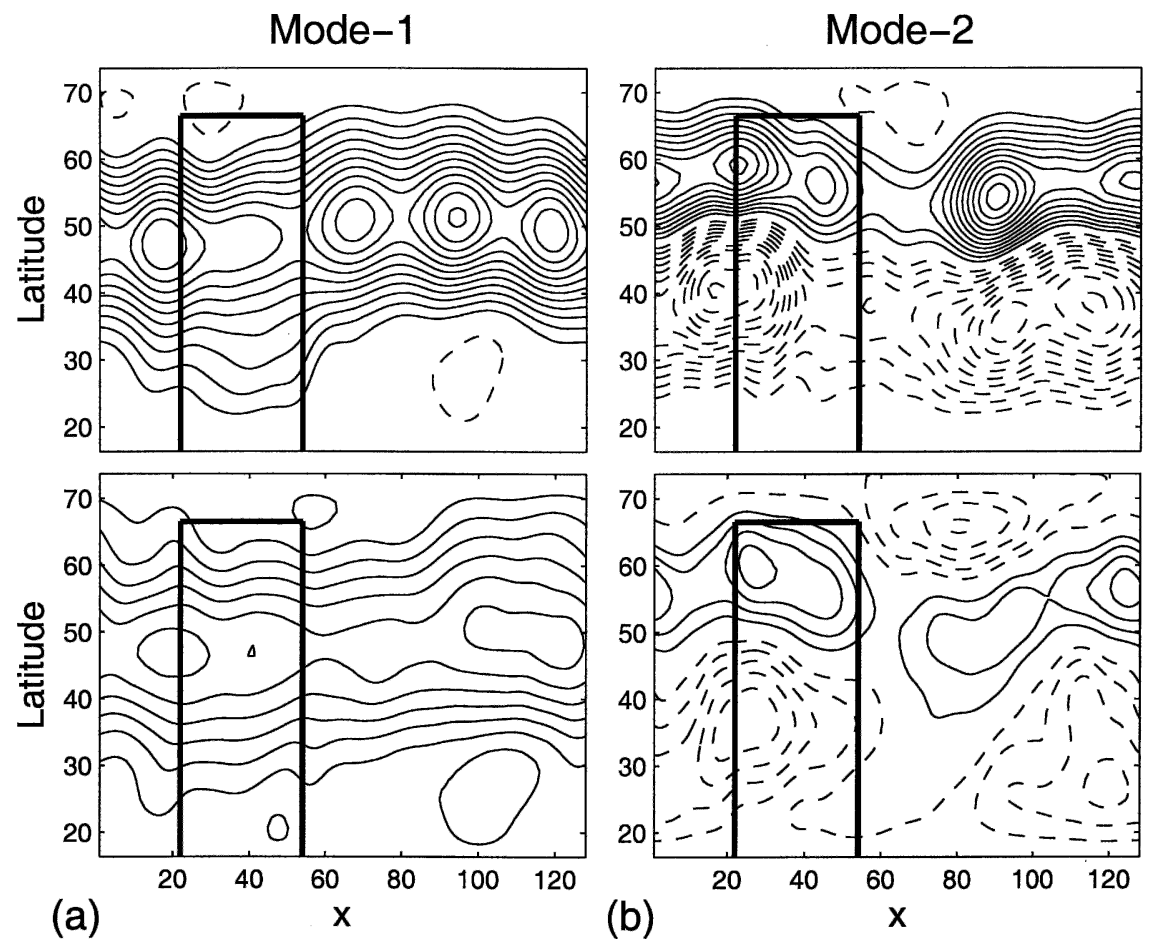

FIG. 7. Leading stationary modes in high-latitude state; $k^{-1}=8.3$ days. (a) EOF $3,10 \%$ (mode 1, see text): (top) upper-layer streamfunction, (bottom) lower-layer streamfunction (CI $\left.=2.5 \times 10^{-4}\right)$; (b) EOF 8, 3\% (mode 2), same conventions and CIs as in (a). Negative contours are dashed, zero contour omitted. Heavy solid lines mark the ocean's coastline.

equivalent barotropic. Waves 5 and 6 (Figs. 8b,d) exhibit a westward tilt with height in the middle of the land region, but become more barotropic as they age and exit the storm track over the ocean. The latter two waves represent the synoptic eddies in our model and account for $12 \%$ and $5 \%$ of total variance, respectively. The 10 leading EOFs we have described account for about $90 \%$ of the model's total variance.

The stationary modes and waves identified above exist in a wide range of $k$ values, for both high-latitude and low-latitude states. The changes in the variance of these modes as a function of $k^{-1}$ are plotted in Fig. 9 , separately for the low-latitude and high-latitude state.

The results for both stationary modes and waves 3 and 4 are shown in Fig. 9a. The behavior of the stationary modes is similar to that of the leading zonally averaged EOFs (see Figs. 4a,b), as expected. In particular, the mode- 1 variance increases significantly for $k^{-1}$ just below the bifurcation point $k^{-1}=5.9$ days. Wave 4 , the dominant mode of variability in the region of bimodality, rapidly loses variance in the unimodal region. The wave-3 variance also decreases fairly rapidly with increasing $k$; its decrease starts at lower $k$ values, so that it has lost already most of its variance before hitting the bifurcation point, while the wave- 5 and wave- 6 variances (Fig. 9b) do not significantly change for $k^{-1}>2$ days.
The bimodality is thus associated with the dynamics of mode 1 and wave 4 , since these are the only two modes that undergo significant changes in the vicinity of the bifurcation. In addition, the choice between the high-latitude and the low-latitude state appears to depend upon the relative amplitude of the wave- 4 and wave- 5 modes, the two leading waves in the model: in particular, wave 4 in the high-latitude state is generally less energetic than its low-latitude analog for sufficiently large $k^{-1}$ (Fig. 9a), while the high-latitude wave 5 is more energetic than its low-latitude counterpart (Fig. 9b).

The stationary modes have most of their power at low frequencies, and do not differ significantly from red noise in this regard (not shown). The power spectra of the waves for the high-latitude state are shown in contours in Fig. 10 as functions of $k$ ( $x$ axis) and frequency ( $y$ axis). The results for the low-latitude state are similar, both qualitatively and quantitatively (not shown). The spectra were computed using Welch's averaged periodogram method: the signal was divided into 128-daylong segments, each of which was detrended, windowed, and then zero padded by an extra length of 64 days on either side; the segments overlap pairwise by one-half of their total length of 256 days. The final spectrum was obtained by averaging over all the periodograms (Oppenheim and Schafer 1989). The shaded regions in all panels are significant at the $95 \%$ level. 


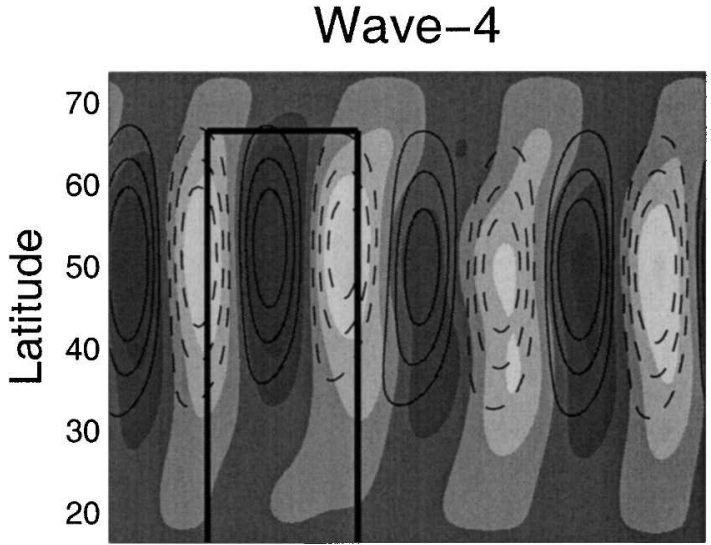

(a)

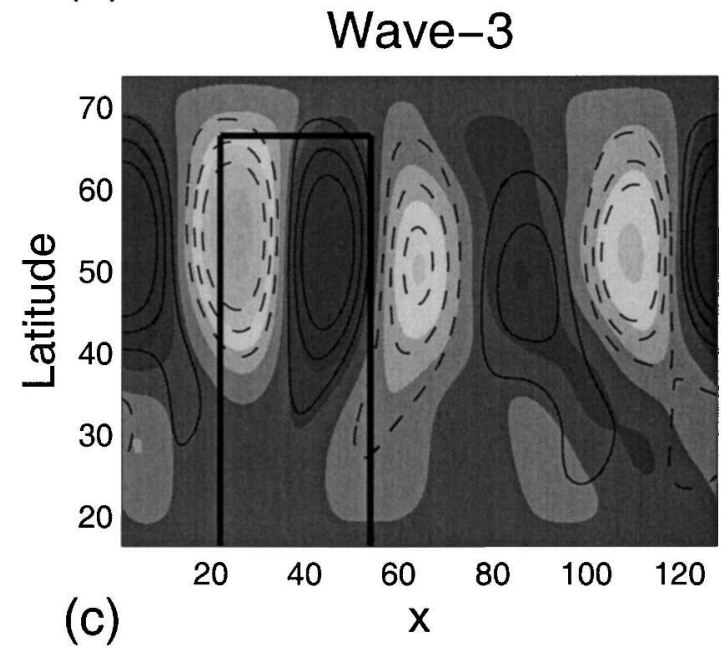

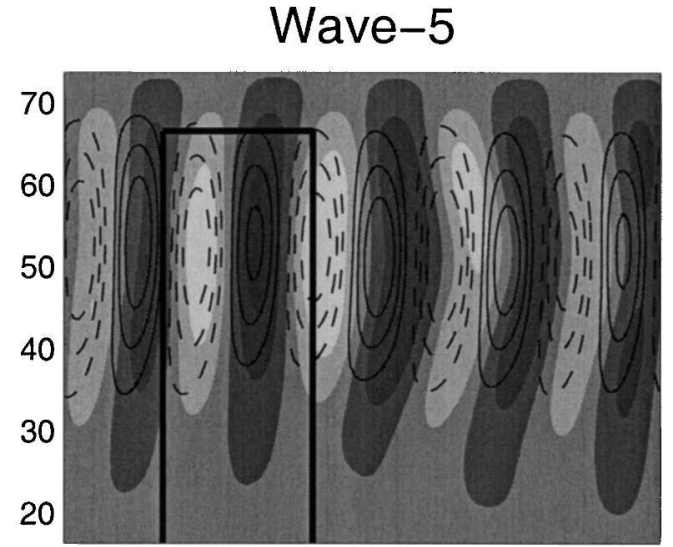

(b)

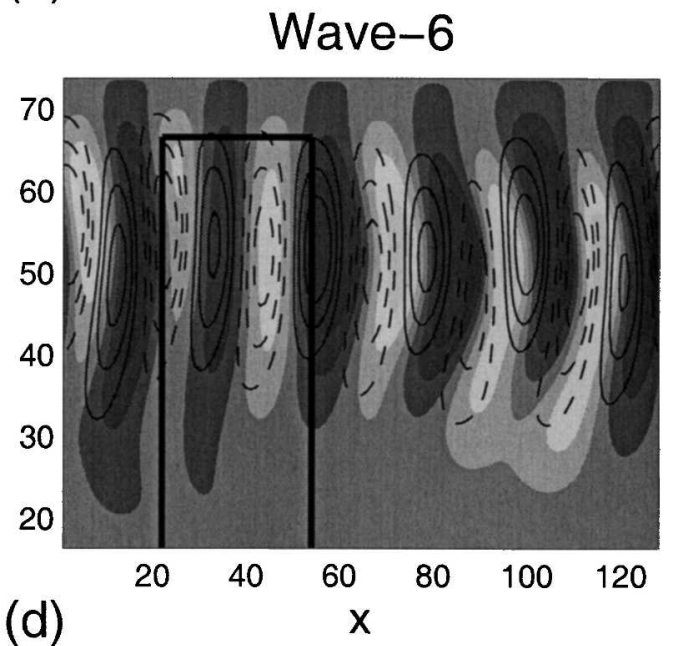

FIG. 8. Leading propagating modes in high-latitude state; $k^{-1}=8.3$ days. (a) EOF $1,50 \%$ (the pair EOF 1-EOF 2 corresponds to wave 4, see text); (b) EOF 4,12\% (the pair EOF 4-EOF 5 corresponds to wave 5); (c) EOF 6, $8 \%$ (the pair EOF 6-EOF 7 corresponds to wave 3); and (d) EOF 9, 5\% (the pair EOF 9-EOF 10 corresponds to wave 6). Lower-layer streamfunction, shading $\left(\mathrm{CI}=5 \times 10^{-4}\right)$; dark shading corresponds to positive values; upper-layer streamfunction $\left(\mathrm{CI}=10^{-3}\right)$; negative contours dashed, zero contour omitted. Heavy solid lines mark the ocean's coastline. The percentages of variance listed correspond to the pair of EOFs involved.

The spectra of the wave modes all exhibit broad peaks that are statistically significant; the heavy solid lines in all the panels will be described in section 5 . Wave 3 has periods of 10 to 15 days, with some dependence on $k^{-1}$. Once again, the behavior of wave 4 changes most strikingly as the bottom drag is decreased: its period increases from about 20 days to longer than 100 days upon approaching the bifurcation point. The periods of waves 5 and 6 increase monotonically with the bottom drag for all $k^{-1}>2$ days, and wave 5 has a lower frequency than wave 6; both periods are generally shorter than 10 days.

We have thus shown that all the modes identified by the fully three-dimensional EOF decomposition of the flow have distinctive properties and are likely to be associated with different dynamical modes.

\section{Vorticity budget considerations}

In this section, we analyze the vorticity budget associated with each of the modes identified in section 4 (section 5a), establish the connection between these statistically determined modes and the system's linear eigenmodes (section 5b), and describe the interactions between these modes and their role in the model's two flow regimes (section 5c).

\section{a. Perturbation-vorticity equations}

We first decompose $\psi$ and $\tau$ as

$$
\psi=\bar{\psi}+\psi^{\prime}, \quad \tau=\bar{\tau}+\tau^{\prime},
$$

where the overbar denotes the time mean and the 

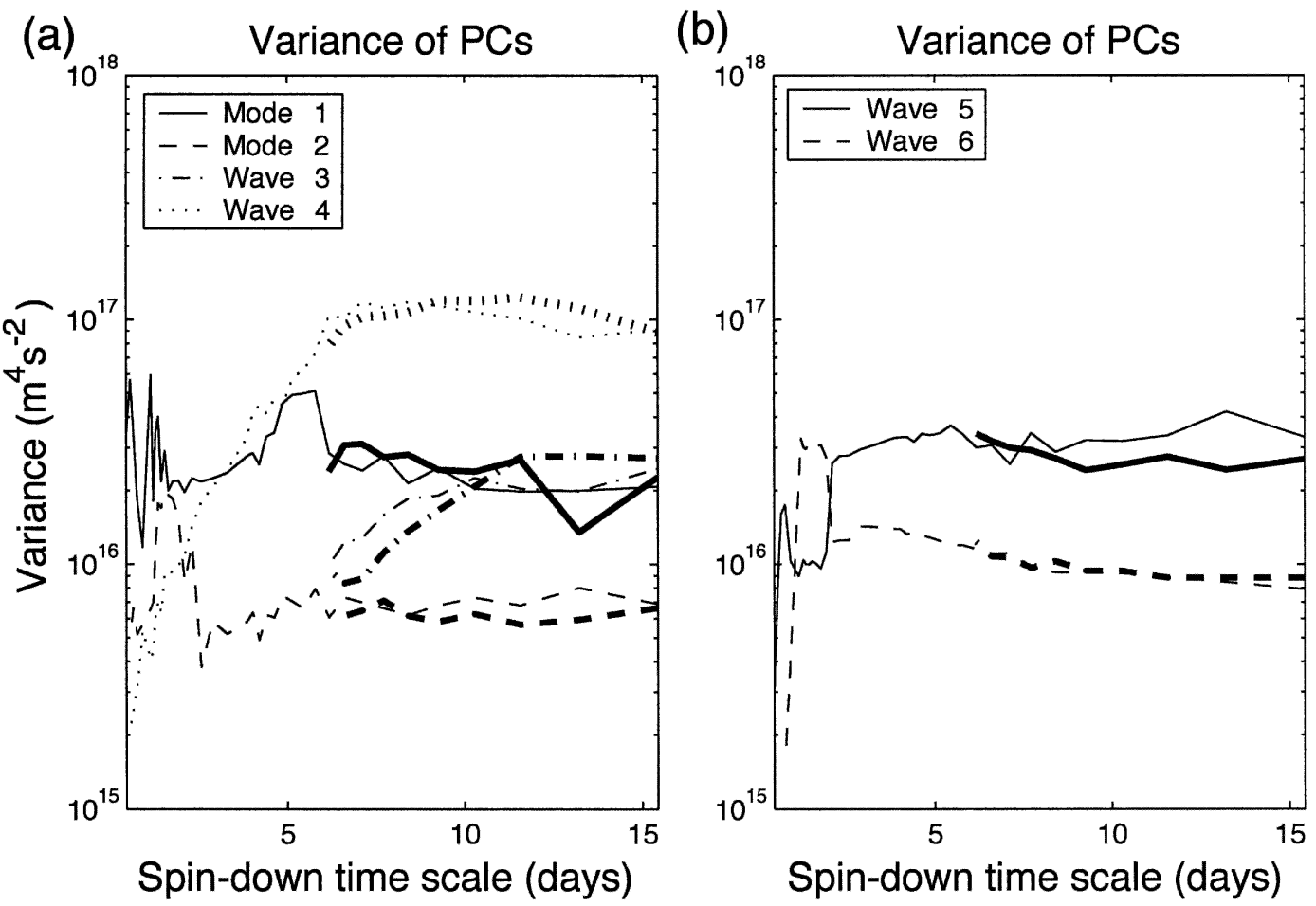

FIG. 9. Dependence of the modes defined in Figs. 7 and 8 on the barotropic spindown time scale $k^{-1}$ (days). (a), (b) Variance of the PCs $\left(\mathrm{m}^{4} \mathrm{~s}^{-2}\right)$ : different lines are described in the legend; heavy lines are for the low-latitude state and light lines for the high-latitude state.

prime a perturbation with respect to it. Next, we substitute the above decomposition into Eq. (A1) and sub- tract from the resulting equation its time mean to get the perturbation vorticity equations

$$
\begin{aligned}
& \frac{\partial q_{\psi}^{\prime}}{\partial t}=-\underbrace{\left[J\left(\bar{\psi}, q_{\psi}^{\prime}\right)+h_{1} h_{2} J\left(\bar{\tau}, q_{\tau}^{\prime}\right)\right]}_{\mathrm{a}}-\underbrace{\left[J\left(\psi^{\prime}, \bar{q}_{\psi}\right)+h_{1} h_{2} J\left(\tau^{\prime}, \bar{q}_{\tau}\right)\right]}_{\mathrm{b}}-\underbrace{\left[k \nabla^{2} \psi^{\prime}+\sum_{n=1}^{3} k^{(n)} \nabla^{2} \psi^{\prime(n)}-A_{H} \nabla^{6} \psi^{\prime}+h_{2} k \nabla^{2} \tau^{\prime}\right]}_{\mathrm{c}} \\
& -\underbrace{\left[J\left(\psi^{\prime}, q_{\psi}^{\prime}\right)+h_{1} h_{2} J\left(\tau^{\prime}, q_{\tau}^{\prime}\right)\right]}_{\mathrm{d}}+\underbrace{\left[\overline{J\left(\psi^{\prime}, q_{\psi}^{\prime}\right)+h_{1} h_{2} J\left(\tau^{\prime}, q_{\tau}^{\prime}\right)}\right]}_{\mathrm{e}}, \\
& \frac{\partial q_{\tau}^{\prime}}{\partial t}=-\underbrace{\left[\left(h_{2}-h_{1}\right) J\left(\bar{\tau}, q_{\tau}^{\prime}\right)+J\left(\bar{\tau}, q_{\psi}^{\prime}\right)+J\left(\bar{\psi}, q_{\tau}^{\prime}\right)\right]}_{\mathrm{a}}-\underbrace{\left[\left(h_{2}-h_{1}\right) J\left(\tau^{\prime}, \bar{q}_{\tau}\right)+J\left(\tau^{\prime}, \bar{q}_{\psi}\right)+J\left(\psi^{\prime}, \bar{q}_{\tau}\right)\right]}_{\mathrm{b}} \\
& +\underbrace{\left[\frac{f_{0}}{H_{a}} \frac{1}{h_{1} h_{2}} F^{\prime}\left(x, y ; \bar{\tau}, \bar{\psi} ; \tau^{\prime}, \psi^{\prime}\right)-\frac{h_{2}}{h_{1}} k \nabla^{2} \tau^{\prime}-\sum_{n=1}^{3} k^{(n)} \nabla^{2} \tau^{\prime(n)}+A_{H} \nabla^{6} \tau^{\prime}-\frac{k}{h_{1}} \nabla^{2} \psi^{\prime}\right]}_{\mathbf{c}} \\
& -\underbrace{\left[\left(h_{2}-h_{1}\right) J\left(\tau^{\prime}, q_{\tau}^{\prime}\right)+J\left(\tau^{\prime}, q_{\psi}^{\prime}\right)+J\left(\psi^{\prime}, q_{\tau}^{\prime}\right)\right]+\frac{f_{0}}{H_{a}} \frac{1}{h_{1} h_{2}} \hat{F}^{\prime}\left(x, y ; \bar{\tau}, \bar{\psi} ; \tau^{\prime}, \psi^{\prime}\right)}_{\mathrm{d}} \\
& +\underbrace{\left.\left[\overline{\left(h_{2}-h_{1}\right) J\left(\tau^{\prime}, q_{\tau}^{\prime}\right)+J\left(\tau^{\prime}, q_{\psi}^{\prime}\right)+J\left(\psi^{\prime}, q_{\tau}^{\prime}\right)}\right]-\frac{f_{0}}{H_{a}} \frac{1}{h_{1} h_{2}} \overline{\hat{F}^{\prime}\left(x, y ; \bar{\tau}, \bar{\psi} ; \tau^{\prime}, \psi^{\prime}\right.}\right)} .
\end{aligned}
$$


(a)

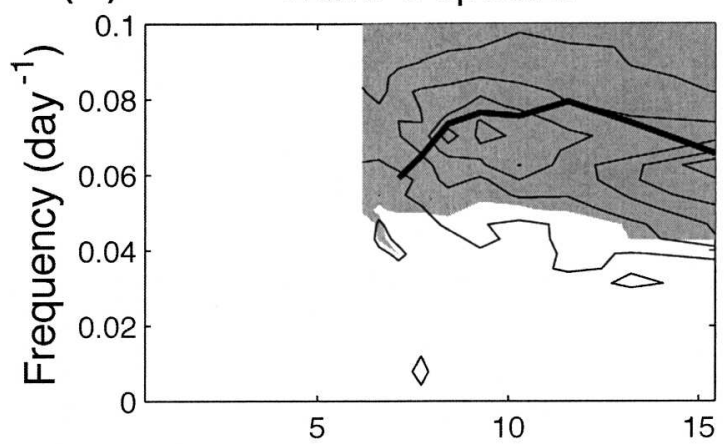

(c)

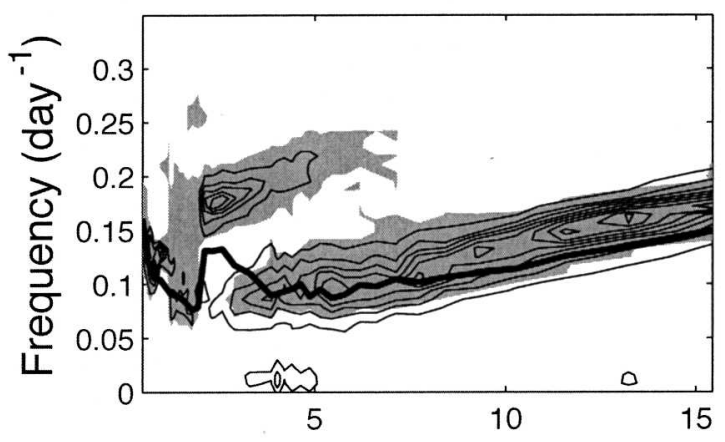

Spin-down time scale (days) (b) Wave-4 spectra

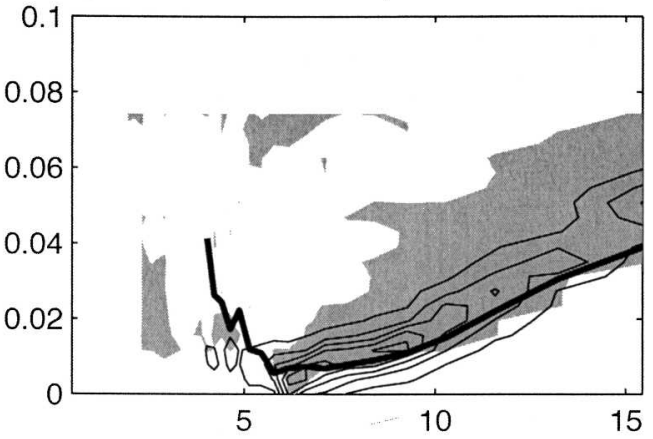

(d) Wave-6 spectra

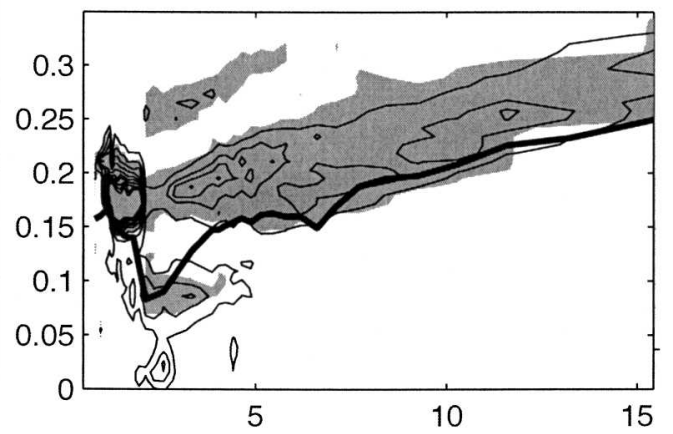

Spin-down time scale (days)

FIG. 10. Power spectra of the propagating modes in the high-latitude state, as a function of the barotropic spindown time scale $k^{-1}$ (days): (a) wave $3(\mathrm{CI}=0.03)$; (b) wave $4(\mathrm{CI}=0.4)$; (c) wave $5(\mathrm{CI}=0.05)$; and (d) wave $6(\mathrm{CI}=0.02)$. Contours are power spectral density $\left(0.78 \times 10^{16} \mathrm{~m}^{4} \mathrm{~s}^{-2} \mathrm{day}^{-1}\right)$; shaded areas are statistically significant at the $95 \%$ level against a null hypothesis of red noise. Heavy solid lines depict the fundamental frequency of the mode as obtained by linear stability analysis (see text).

Terms (a) and (b) represent the advection of perturbation vorticity by the climatological flow and the advection of climatological vorticity by the perturbation flow, respectively; (c) are linear damping terms; (d) are terms that are nonlinear in $\psi^{\prime}$ and $\tau^{\prime}$; and (e) are the clima- tological values of these nonlinear terms. The notation $\hat{F}^{\prime}$ is used to denote the part of the model's thermal forcing $F$ [see Eq. (A1b) and appendix A] that is nonlinear in $\psi^{\prime}$ and $\tau^{\prime}$.

The climatological vorticity balance reads as follows:

$$
\begin{aligned}
0= & -J\left(\bar{\psi}, \bar{q}_{\psi}\right)-h_{1} h_{2} J\left(\bar{\tau}, \bar{q}_{\tau}\right)-k \nabla^{2} \bar{\psi}-\sum_{n=1}^{3} k^{(n)} \nabla^{2} \bar{\psi}^{(n)}+A_{H} \nabla^{6} \bar{\psi}-h_{2} k \nabla^{2} \bar{\tau}-\left[\overline{J\left(\psi^{\prime}, q_{\psi}^{\prime}\right)+h_{1} h_{2} J\left(\tau^{\prime}, q_{\tau}^{\prime}\right)}\right], \\
0= & -\left(h_{2}-h_{1}\right) J\left(\bar{\tau}, \bar{q}_{\tau}\right)-J\left(\bar{\tau}, \bar{q}_{\psi}\right)-J\left(\bar{\psi}, \bar{q}_{\tau}\right)+\frac{f_{0}}{H_{a}} \frac{1}{h_{1} h_{2}} \bar{F}(x, y ; \bar{\tau}, \bar{\psi})-\frac{h_{2}}{h_{1}} k \nabla^{2} \bar{\tau}-\sum_{n=1}^{3} k^{(n)} \nabla^{2} \bar{\tau}^{(n)}+A_{H} \nabla^{6} \bar{\tau}-\frac{k}{h_{1}} \nabla^{2} \bar{\psi} \\
& -\left[\overline{\left(h_{2}-h_{1}\right) J\left(\tau^{\prime}, q_{\tau}^{\prime}\right)+J\left(\tau^{\prime}, q_{\psi}^{\prime}\right)+J\left(\psi^{\prime}, q_{\tau}^{\prime}\right)}-\frac{f_{0}}{H_{a}} \frac{1}{h_{1} h_{2}} \overline{\hat{F}^{\prime}}\left(x, y ; \bar{\tau}, \bar{\psi} ; \tau^{\prime}, \psi^{\prime}\right)\right] .
\end{aligned}
$$

The last term in square brackets in each of the Eqs. (3a) and $(3 b)$ is due to the time-mean effect of nonlinear eddy interactions. These terms also appear with negative sign as terms (e) in the perturbation Eqs. (2a) and (2b).

\section{b. Analysis of the linearized equations}

The linearized perturbation vorticity equations are obtained by neglecting terms (d) and (e) in Eq. (2). 
First, we substitute the spatial fields associated with the stationary modes 1 and 2 (see section 4 ) into the linearized Eqs. (2a) and (2b), and compute the tendencies associated with each of the terms (a), (b), and (c). The term (c) represents linear damping of each mode and is, therefore, not very informative. In contrast, terms (a) and (b) have an interesting property: their sum is much less than each of the individual components, while the latter are comparable in magnitude. This property is illustrated in Fig. 11.

In Fig. 11a, we plot, for modes 1 and 2, the ratio of the spatial norms $r \equiv[|(a)+(b)|] / \min [|(a)|,|(b)|]$ in the linearized Eq. (2a), together with the correlation coefficient between (a) and (b), as a function of $k^{-1}$. The quantitative results for Eq. (2b) (not shown) are the same. The spatial correlation between (a) and (b) is close to -1 , while $r$ is generally less than 0.2 ; the term (a) + (b) is nearly uncorrelated with (a) and (b) (not shown). A somewhat less significant cancellation is seen for low-latitude mode-2 tendencies, where the correlation is around -0.9 and $r \approx 0.4$. This behavior may be associated with the fact that mode 2 , having a relatively small variance (see Fig. 9a), is contaminated by spatial structures associated with different dynamical modes.

It thus appears that modes 1 and 2 are associated with stationary Rossby waves; the type of cancellation identified above is a characteristic feature of such waves. We now argue that the propagating modes are, in turn, associated with propagating Rossby waves. To do so, we demonstrate that these empirical modes correspond to the propagating linear eigenmodes of the linearized perturbation-vorticity Eq. (2).

First, we compute the tendencies $\partial \Psi_{1} / \partial t$ and $\partial \Psi_{2} / \partial t$ of the linearized Eq. (2) that are associated with the members of the wave pair $\Psi_{1} \equiv\left[\psi_{1}, \tau_{1}\right]$ and $\Psi_{2} \equiv\left[\psi_{2}, \tau_{2}\right]$, where indices 1 and 2 denote the two members of the pair. We then find the coefficients $a_{i j}, i, j=1,2$ that minimize the quantities $\varepsilon_{i}, i=1,2$ in

$$
\begin{aligned}
& \frac{\partial \Psi_{1}}{\partial t}=a_{11} \Psi_{1}+a_{12} \Psi_{2}+\varepsilon_{1} \Delta \Psi_{1}, \\
& \frac{\partial \Psi_{2}}{\partial t}=a_{21} \Psi_{1}+a_{22} \Psi_{2}+\varepsilon_{2} \Delta \Psi_{2} ;
\end{aligned}
$$

here, the wave pair tendencies are normalized by their respective standard deviations, $\Delta \Psi_{1}$ and $\Delta \Psi_{2}$ are both residuals that have unit standard deviation and are uncorrelated with the wave pair fields and tendencies. This minimization problem is solved by least squares.

We define the least squares fit to be successful if $\max \left(\varepsilon_{1}, \varepsilon_{2}\right)<0.2$. For the successful fits, we can compute the periods and growth rates associated with each of the wave modes, as the eigenvalues of the matrix $\mathbf{A}$ $\equiv\left(a_{i j}\right)$. The periods we have obtained by this procedure are superimposed as heavy solid lines on the spectra in Fig. 10 and they match well the major spectral peaks shown in this figure. This good match confirms that the wave modes identified by the PC analysis of section 4 do indeed correspond to propagating eigenmodes of the linearized perturbation-vorticity Eq. (2).

The growth rates of all waves, plotted in Figs. 11b,c, increase with bottom drag (see also James and Gray 1986), except for wave 3; the latter is equivalent barotropic and there is no mechanism to counteract the damping effect of increasing bottom friction. Baroclinic waves 5 and 6 have growth rates that increase monotonically with bottom drag (Fig. 11c), while their variance stays approximately constant (Fig. 9b). This discrepancy implies that energy is extracted nonlinearly from these waves with increasing efficiency as the bottom drag increases.

\section{c. Nonlinear eddy effects}

To gain more insight into how wave-mean flow interactions in our model result in bimodal behavior, we now consider the role of eddies in maintaining a given high-latitude or low-latitude state. The time-mean eddy forcing is given by the last terms in Eqs. (3a) and (3b):

$$
\begin{aligned}
F_{\psi}= & -\left[\overline{J\left(\psi^{\prime}, q_{\psi}^{\prime}\right)+h_{1} h_{2} J\left(\tau^{\prime}, q_{\tau}^{\prime}\right)}\right], \\
F_{\tau}= & -\left[\overline{\left(h_{2}-h_{1}\right) J\left(\tau^{\prime}, q_{\tau}^{\prime}\right)+J\left(\tau^{\prime}, q_{\psi}^{\prime}\right)+J\left(\psi^{\prime}, q_{\tau}^{\prime}\right)}\right. \\
& \left.-\frac{f_{0}}{H_{a}} \frac{1}{h_{1} h_{2}} \overline{\hat{F}^{\prime}\left(x, y ; \bar{\tau}, \bar{\psi} ; \tau^{\prime}, \psi^{\prime}\right)}\right] .
\end{aligned}
$$

The last term in Eq. (5b) is small, so that the main nonlinear eddy effects in the model are associated with the vorticity advection terms. Because of the time averaging present in Eq. (5) and the orthogonality of the PCs in time, the full climatological eddy forcing $\left\{F_{\psi}, F_{\tau}\right\}$ can be decomposed into a sum of terms, each of which represents the contribution of an individual mode.

The eddy forcing associated with stationary modes 1 and 2 is shown in Fig. 12. The self-interaction of these modes is extremely small, so that their contribution to the total eddy forcing is negligible. These stationary Rossby waves are thus close to the free modes of the system in that they do not exchange energy with the mean flow. The two modes' spatial pattern is therefore selected by nonlinearity in a way that minimizes their effective damping. At low frequencies, the other modes are strongly damped by nonlinear effects, while the free modes are not, which helps explain the dominance of the latter. At shorter time scales, however, the stationary and propagating modes do interact. For example, the dominance of mode 1 over mode 2 (see again Fig. 4) may be due to differences in the way the two modes get energized by shorter, propagating waves (Robinson 1996, 2000; Kravtsov et al. 2003).

The time-mean, zonally averaged eddy forcing associated with propagating waves is shown in Fig. 13. The eddy forcing by wave 3 and wave 4 (Figs. 13a,b and Figs. 13c,d) tends to reduce the jet velocity near its axis and enhance the mean zonal velocity on the flanks of the jet: this leads, presumably, to meridional migrations 


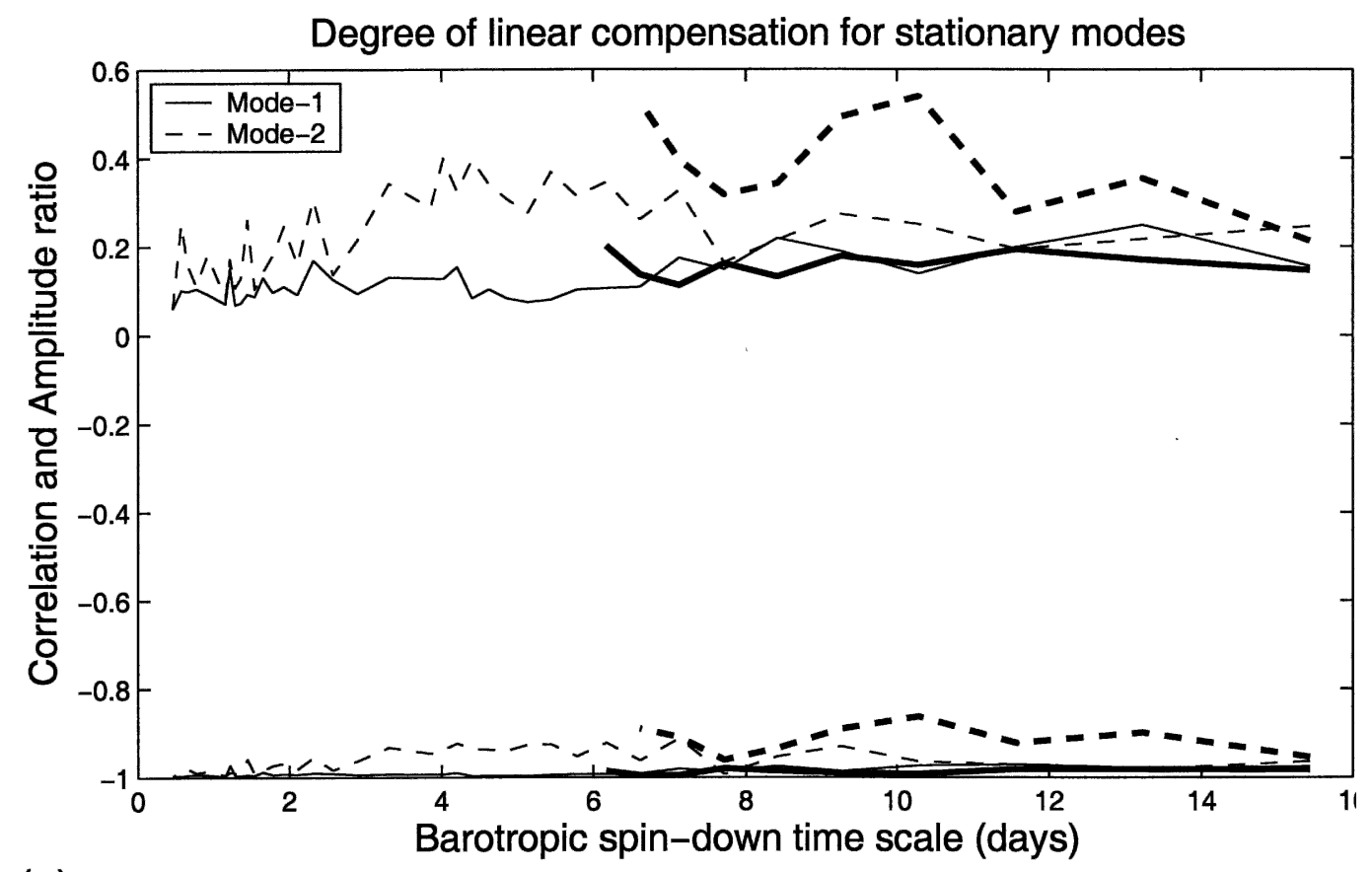

(a)

\section{Growth rate}

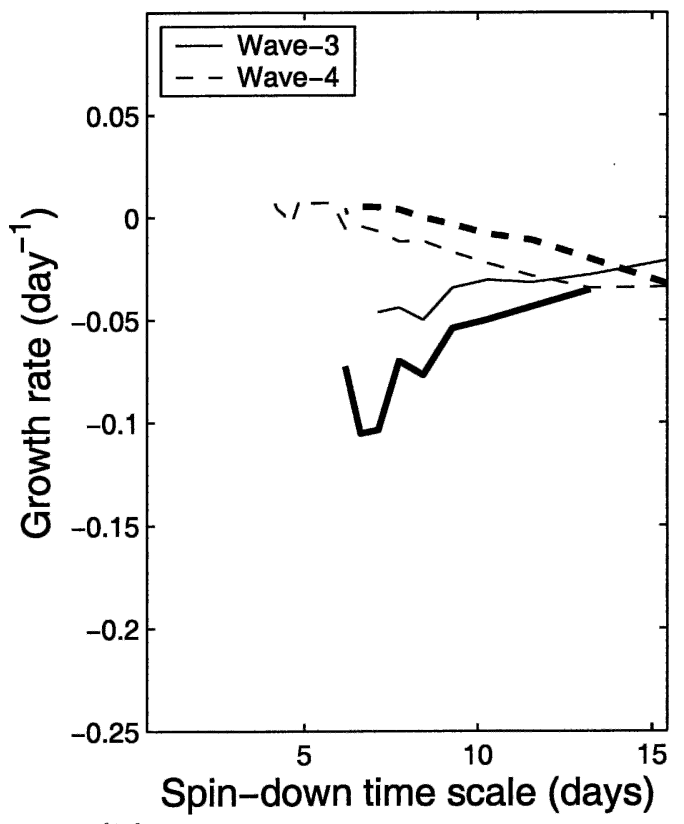

(b)

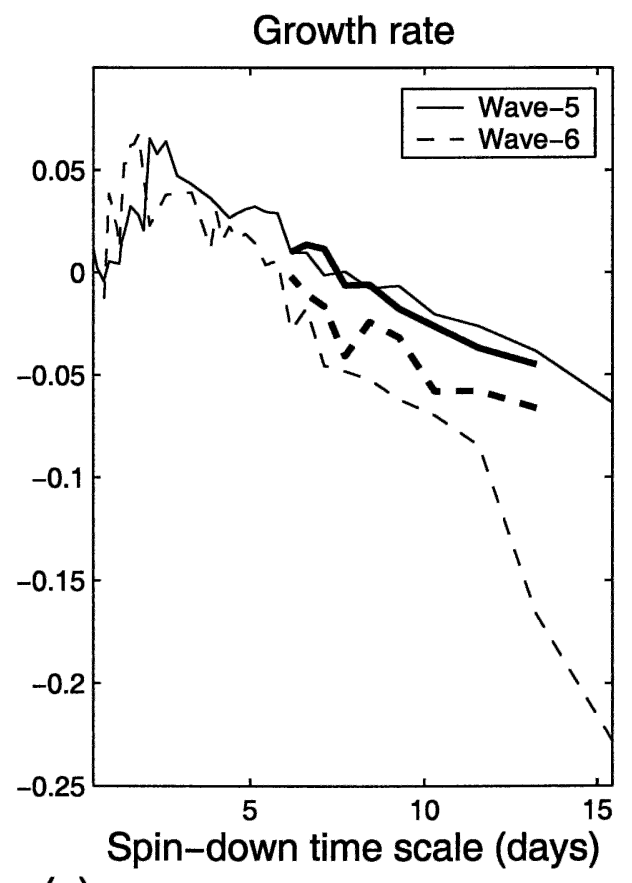

(c)

FIG. 11. Results of linear vorticity budget analysis, as a function of the barotropic spindown time scale $k^{-1}$ (days). (a) Degree of linear compensation for stationary modes. Lines in the upper, positive portion of the graph show the ratio of the spatial standard deviation of the total barotropic tendency due to the advection operator, linearized about climatology, and the sum of the standard deviations of the two terms that comprise this operator [terms (a) and (b) of Eq. (4a)]; lines that have negative $y$ values show the spatial correlation between the two parts of the total advective tendency. (b), (c) Growth rates of wave modes. In each panel, the lines are identified in the legend; heavy lines show the results for the low-latitude state and light lines for the high-latitude state. 
(a) Mode-1 dU/dt (Cl=0.02)

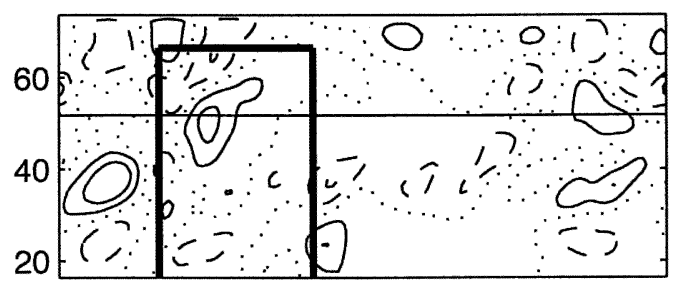

(b) Mode-2 dU/dt $(\mathrm{Cl}=0.02)$

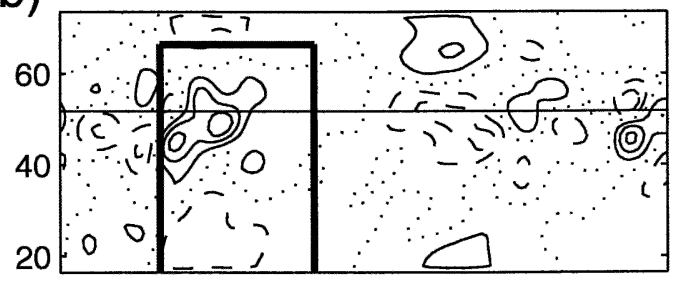

Mode $-1 \mathrm{dT} / \mathrm{dt}(\mathrm{Cl}=0.02)$

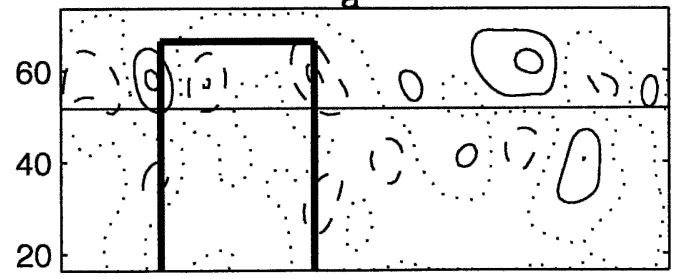

Mode-2 dT a $/ \mathrm{dt}(\mathrm{Cl}=0.02)$

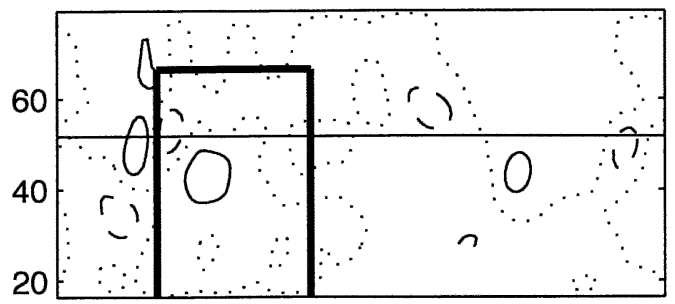

FIG. 12. Effect of the stationary eddies on the maintenance of the climatological jet (see text): (a) mode 1, and (b) mode 2. Results are shown for the high-latitude state at $k^{-1}=8.3$ days. (left) Barotropic zonal velocity tendency $\left(\mathrm{m} \mathrm{s}^{-1} \mathrm{day}^{-1}\right)$; (right) atmospheric temperature tendency $\left({ }^{\circ} \mathrm{C} \mathrm{day}^{-1}\right)$. For each panel, $\mathrm{CI}=0.02$ units, negative contours dashed, zero contour dotted. Heavy solid lines mark the ocean basin's coastline; the light solid horizontal line marks the climatological jet position.

of the jet. Another important wave-4 effect is to maintain the atmospheric temperature gradient in the jet region (Fig. 13d). Waves 5 and 6 (Figs. 13e,f and Figs. $13 \mathrm{~g}, \mathrm{~h})$ tend to make the jet narrower and stronger; they also tend to flatten the atmospheric temperature profile.

Recall that the eddies were defined here as anomalies about a given high-latitude or low-latitude branch of model solutions (see section $3 b$ ). The action of these eddies on maintaining or disrupting either regime thus helps explain the differences between the two regimes' persistence in a free integration, in which transitions between the two states are allowed (see again section $3 \mathrm{c}$ and Fig. 5). In particular, low-latitude wave 4 is more energetic, and low-latitude wave 5 is less energetic than its high-latitude counterpart, respectively (Figs. 9a,b and Figs. 11b,c).

A major nonlinear effect of baroclinic wave 5 is to reduce the meridional temperature gradient (see Fig. 13f). Since the low-latitude wave 5 has smaller variance, this effect is less pronounced than for the high-latitude state; therefore, the meridional temperature gradient across the jet in the low-latitude state is larger than in the high-latitude state (not shown). By thermal wind balance, this corresponds to a stronger low-latitude jet (see Fig. 3b). Wave 4, which is nearly equivalent barotropic and extracts its energy from the climatological jet, will then have a larger variance in the low-latitude state. The nonlinear effect of this wave on climatology is destabilizing (see Fig. 13c) and it is likely to result in meridional migrations of the jet. Therefore, the transition from the low-latitude state to the high-latitude state is more likely than the reverse transition, and so the high-latitude state is preferred and more persistent in this model (see Fig. 5).

As mentioned in section $4 \mathrm{~b}$, bimodality in this model involves interactions between mode 1 and wave 4 . To recapitulate, the reasoning behind this statement is the following. First, mode 1 and wave 4 are the only two modes whose variances change significantly when crossing the bifurcation point (Fig. 9a). Second, the power spectra of wave 4 show a persistent decrease of frequency as $k$ is increasing to its critical value (Fig. $10 \mathrm{~b})$, where the dominant frequency of wave 4 becomes very low; hence, the interaction of wave 4 with mode 1 , whose temporal behavior resembles red noise, is likely to become increasingly important there. Last, but not least, the largely zonal mode 1 possesses a wave- 4 modulation (Fig. 7a).

\section{Concluding remarks}

\section{a. Summary}

We have investigated the behavior of a two-layer, quasigeostrophic (QG), midlatitude atmospheric channel model with flat bottom, subject to zonally inhomogeneous thermal forcing (section 2, Fig. 1). The model's evolution has been studied in a wide range of the bottom-drag parameter $k$. For $k^{-1}>1$ day, the model's climatology and variability is dominated by a narrow jet that is only slightly modulated zonally due to the imposed land-sea thermal contrast (sections 3 and 4, Figs. 2, 6, and 7).

The model's zonal-mean flow is bimodal in a realistic range of the spindown time scale (Lorenz and Hartmann 2001, 2003) of 6 days $<k^{-1}<10$ days: two dif- 
(a)

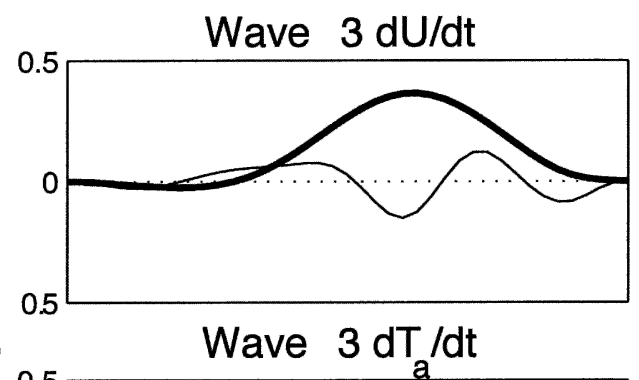

(b)

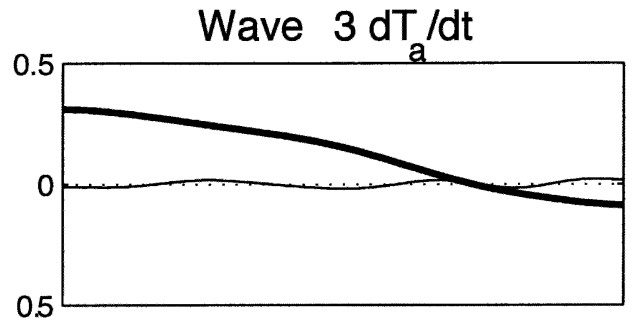

(e)

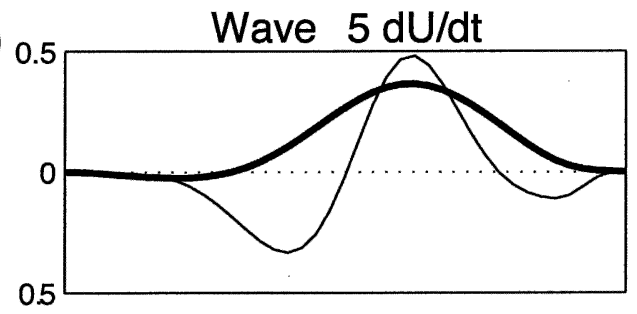

(f)

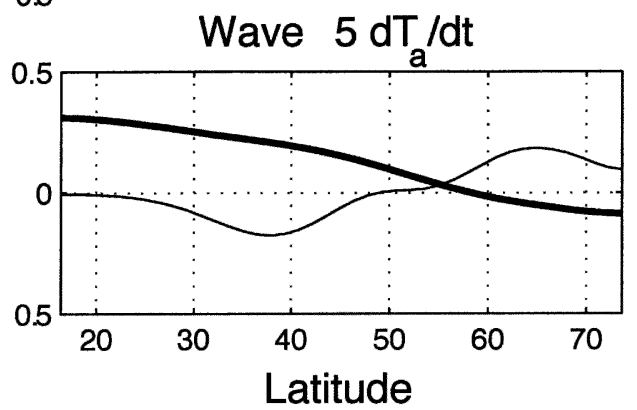

(c)

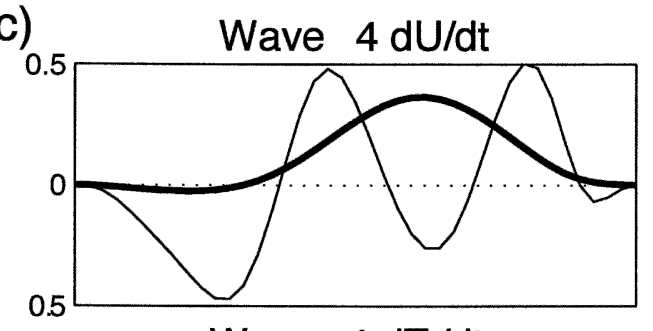

(d)

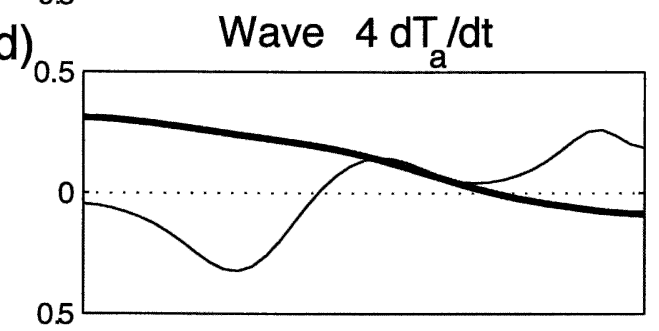

\section{(g)}

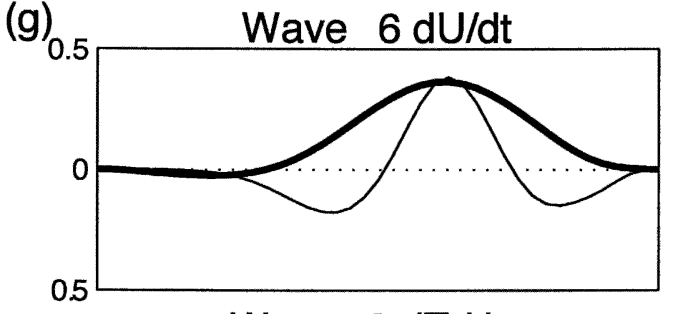

(h)

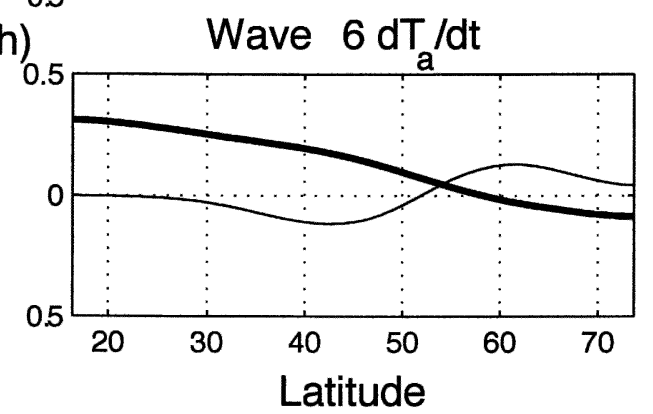

FIG. 13. As in Fig. 12 (except for placement of panels) but for the zonally averaged tendencies associated with the leading propagating modes (light solid lines). Heavy solid lines show climatological, zonally averaged barotropic zonal velocity divided by $50\left(\mathrm{~m} \mathrm{~s}^{-1}\right)$, and zonally averaged atmospheric temperature $\left({ }^{\circ} \mathrm{C}\right)$ divided by 100 .

ferent regimes are characterized by the position of the jet, which is shifted poleward or equatorward of the channel axis (Fig. 3a). Irregular transitions between these two states dominate the model's variability: the high-latitude state is more persistent than the lowlatitude state (Fig. 5). The leading low-frequency mode of the system's variability, mode 1 , is associated with meridional shifts of the jet, while a less energetic mode 2 describes changes in the jet's intensity (Figs. 2a and 7).

The stationary modes 1 and 2, as well as the leading propagating waves (Fig. 8) obtained by PC analysis, correspond to eigenmodes obtained by linearization about each regime's climatology, separately (Figs. 10 and 11). This close correspondence between EOFs and linear eigenmodes reminds us of Brunet's (1994) empirical normal modes and we plan to explore the connection, if any, in future work.
The self-interactions of modes 1 and 2 are negligibly small (Fig. 12). These stationary modes thus appear, to a good approximation, as free modes, since they do not exchange energy with the time-mean flow, while they dominate model behavior at low frequencies, where all other modes are nonlinearly damped. The dominance of mode 1 over mode 2 may be due to the different ways in which the two modes are energized by the higher-frequency eddies.

The high-latitude and low-latitude jet regimes are shaped by the interaction of the latitudinally varying waves (Fig. 8): the effect of baroclinic wave-wave interactions, associated with our model's synoptic eddies, is merely to maintain a narrow and intense highlatitude or low-latitude jet (Fig. 13). In contrast, an external Rossby wave 4, with its nearly equivalent barotropic structure, tends to disrupt a given jet state by 
inducing transitions to the other state. The choice of wave 4 for this transition-inducing role might be related to our model geometry. We suspect, however, that a similar role will be played by a possibly different external Rossby wave in a model that mimics more faithfully lower boundary conditions in the $\mathrm{NH}$ or $\mathrm{SH}$ flow.

This behavior helps explain the differences between high-latitude and low-latitude states. In the low-latitude state, synoptic eddies are less intense. This leads to an increase of the north-south temperature gradient and, by thermal wind balance, to a more intense jet. More energy is thus available to feed the variability of external Rossby wave 4, which destabilizes this state's climatological jet. This destabilization is reinforced by the reduced contribution of the synoptic eddies to maintaining the jet. Therefore, the low-latitude state is nonlinearly less stable compared to the high-latitude state; the latter is thus preferred by the system.

Synoptic eddies play therewith an important role in determining the relative persistence of the two states. The model's bimodality, however, is primarily due to interactions between mode 1 and a wave 4; these are the only two modes that undergo considerable changes as the bifurcation point is crossed (Figs. 9 and 10). Close to this point, wave-4 frequency becomes very low (Fig. 10b) and its interaction with low-frequency mode 1 becomes critically important there. The spatial signatures of this interaction are also manifest in the wavenumber-4 modulation of the predominant zonal symmetry of mode 1 (Fig. 7a).

Our model's nonlinear dynamics is thus dominated by certain types of wave-wave and wave-mean flow interactions that involve only a small number of modes across a wide range of spindown time scales. This interpretation is supported by the increase of variance of the zonal-flow PCs 1 and 2 near the numerically inferred bifurcation point at $k^{-1}=5.9$ days (see Fig. 4a). The two leading zonal mean flow EOFs capture the essential difference between the high-latitude and lowlatitude regimes, with respect to the position (EOF 1) and the intensity (EOF 2) of the jet. A linear combination of the two is therefore quite likely to approximate well the eigenmode whose change of stability gives rise to the bifurcation. Near the bifurcation, the higher-frequency, low-variance modes of variability act as internal system noise and "pump up" the variability of the stationary, high-variance modes. This scenario is entirely consistent with the expected behavior of nonlinear, stochastically forced systems (Schuss 1980; Gardiner 1983).

\section{b. Discussion}

The dominant LFV modes in our model are related to the annular modes observed in the atmosphere (see section 1). Previous work (Branstator 1992; Metz 1994; Da Costa and Vautard 1997; Itoh and Kimoto 1999; Kimoto et al. 2001; Kravtsov et al. 2003) had already found model stationary modes that correspond to eigenmodes of the system linearized about its climatology. In the present study, the linearization was carried out about the climatology of each regime separately, and the association of the stationary modes with these regime-specific eigenmodes is much closer. Moreover, these modes are associated with nonlinear free modes of the system in that they do not exchange energy with the time-mean flow; this nonlinear selection explains their dominance at low frequencies. The importance of such free modes in atmospheric LFV has been discussed by Branstator and Opsteegh (1989) and Marshall and So (1990), while Greatbatch $(1987,1988)$ and Ghil et al. (2002) discussed their role in wind-driven ocean dynamics. Since the self-interaction of the free modes vanishes, linear stochastic models might be quite adequate for the quantitative description of the system's low-frequency behavior away from the region of bimodality (Kidson and Watterson 1999; Feldstein 2000). Even in this unimodal region, however, the quantitative success of such models does not mean that the underlying dynamics is indeed linear.

We have shown that not only stationary modes, but also leading propagating waves are associated with the eigenmodes of our model's linearized operator. A similar conclusion was implicit in the earlier work of Kravtsov et al. (2003). Our present computations, however, show the dynamical significance of linear modes more explicitly and identify particular eigenmodes that are most important for the model's behavior. These results should be compared with those of Farrell and Ioannou (1993, 1995), who studied finite-time growth of linear perturbations around sheared background flows.

Bimodality enters our model's behavior when the bottom drag becomes sufficiently small $\left(k^{-1}>5.9\right.$ days). We tracked the two equilibria by introducing a small correction term that prevents regime transitions (section 3). Without this term, the model behavior for moderate bottom-drag values, $5.9<k^{-1}<10$ days, consists of irregular shifts between the two states that are characterized by the latitude and intensity of the jet. S. Koo and colleagues identified this type of behavior in SH observations (Koo et al. 2003) and in their primitive equation model (Koo 2002) by constructing composites of the persistent anomalies with respect to climatology and tracking transitions between them.

Thorncroft et al. (1993) have described two different types of the synoptic eddy life cycles. Akahori and Yoden (1997) then found that each of these life cycle types is preferentially associated with either high- or lowlatitude jet states in their primitive equation model; moreover, they showed preference for one regime or the other, depending on the value of the bottom drag. Our results are consistent with these findings, but go further in terms of explaining the mechanisms that give rise to the bimodality.

The eddies help maintain both the high-latitude and low-latitude equilibria, as shown by Koo and Ghil (2002) in a highly truncated baroclinic model. Our 
model has a much higher resolution, so that we are able to distinguish between various wave processes and determine their respective roles in the system's bimodal behavior. In particular, we found that the bimodality is due to interactions between stationary and propagating modes that are nearly equivalent barotropic (see also Kravtsov et al. 2003). The synoptic eddy effects are not crucial for the existence of the two states, but play a role in determining the preferred state. The existence of a preferred state, which is characterized by a more concentrated jet and enhanced synoptic eddy activity, is conceptually consistent with observational results for zonal-flow vacillation (Hartmann 1995).

The role of synoptic eddies in the unimodal region $\left(k^{-1}<5.9\right.$ days) is not fully understood (Robinson 1996, 2000; Kravtsov et al. 2003). They seem to play a role in determining the relative variances of mode 1 and mode 2 (Feldstein and Lee 1998; Lorenz and Hartmann 2001, 2003). Robinson $(1996,2000)$ argued that the socalled synoptic eddy feedback, that is anomalous generation of synoptic eddies in certain phases of lowfrequency evolution, is a major factor in selecting mode 1 to be dominant for high bottom drag. In contrast, Kravtsov et al. (2003) showed that this dominance is consistent with passive steering of synoptic eddies by LFV. We did not find here significant differences in the way synoptic eddies affect the low-frequency flow for either low or moderate bottom-drag values; our results are thus consistent with those of Kravtsov et al. (2003).

We do find the synoptic eddy feedback to be important only for very high and unrealistic values of bottom drag (see appendix B); the strongly nonlinear relaxation oscillation that characterizes model behavior at such values relies on modifications of the mean flow by the synoptic eddies that favor the reinforced generation of these eddies. This specific physical mechanism is associated with the presence of a homoclinic orbit in our high-order system's phase space. The details of system behavior in this transition-to-chaos region do not seem to play a significant role in the more turbulent and realistic regimes obtained at lower values of the bottom drag. Still, a small number of modes dominate model behavior in the latter regimes as well (see sections 4 and 5).

Its flat bottom and the slight degree of zonal asymmetry in its climate makes our model's behavior more relevant to that found in $\mathrm{SH}$ observations. The rel- evance of these results to NH LFV is still a matter of debate (see, e.g., Wallace 2000; Ghil and Robertson 2002). The role of topography in NH LFV has been explored in a sequence of papers (Charney and DeVore 1979; Pedlosky 1981; Legras and Ghil 1985; Ghil and Robertson 2000, and references therein). These theories rely on a combination of topographic resonance and barotropic instability. In contrast, our flat-bottom model's quasi-stationary waves are weak and barotropic instability is not very efficient in exciting significant variability without stochastic energy input from synoptic eddies (see Swanson 2000).

According to Lorenz and Hartmann (2001, 2003), the spindown time scale for the $\mathrm{NH}$ is equal to $k^{-1} \approx 7$ days, and it is $k^{-1} \approx 9$ days in the $\mathrm{SH}$. Our model diagnostic thus imply that both hemispheres are likely to be in a bimodal regime $\left(6<k^{-1}<10\right.$ day) and quite close to the point of bifurcation to the coexistence of two regimes. If so, the behavior in both hemispheres may involve ultralow-frequency, external Rossby waves. Koo et al.'s (2003) observational results indeed show not only the presence of bimodality in the $\mathrm{SH}$ zonal-mean zonal flow, but also the existence of an equivalent barotropic oscillatory mode with a period of 135 days. We have obtained similar observational results for the NH zonal-mean zonal flow (S. Kravtsov, A. W. Robertson, and M. Ghil 2005, unpublished manuscript). Successful prediction of observed multiple regimes and low-frequency oscillations by our simple model argues that it has captured essential aspects of annular-mode dynamics in both hemispheres.

Acknowledgments. It is a pleasure to acknowledge useful discussions and correspondence concerning Fig. 4 with G. Nicolis and A. Sutera. We also thank two anonymous reviewers for their comments, which helped improve the presentation. This research was supported by NSF Grants ATM-00-82131 and OCE-02221066 (MG and SK) and DOE Grant DE-FG-0301ER63260 (AWR and SK).

\section{APPENDIX A}

\section{Governing Equations}

The equations for the barotropic component $\psi$ and baroclinic component $\tau$ of the streamfunction are

$$
\begin{aligned}
\frac{\partial q_{\psi}}{\partial t}+J\left(\psi, q_{\psi}\right)= & -k \nabla^{2} \psi-\sum_{n=1}^{3} k^{(n)} \nabla^{2} \psi^{(n)}+A_{H} \nabla^{6} \psi-h_{1} h_{2}\left[J\left(\tau, q_{\tau}\right)+\frac{k}{h_{1}} \nabla^{2} \tau\right], \\
\frac{\partial q_{\tau}}{\partial t}+\left(h_{2}-h_{1}\right) J\left(\tau, q_{\tau}\right)= & \frac{f_{0}}{H_{a}} \frac{1}{h_{1} h_{2}} F(x, y ; \tau, \psi)-\frac{h_{2}}{h_{1}} k \nabla^{2} \tau-\sum_{n=1}^{3} k^{(n)} \nabla^{2} \tau^{(n)}+A_{H} \nabla^{6} \tau \\
& -\left[J\left(\tau, q_{\psi}\right)+J\left(\psi, q_{\tau}\right)+\frac{k}{h_{1}} \nabla^{2} \psi\right] .
\end{aligned}
$$


Here

$$
q_{\psi}=\nabla^{2} \psi+\beta y, \quad q_{\tau}=\nabla^{2} \tau-\frac{1}{R_{d}^{2}} \tau
$$

are the barotropic and baroclinic component of the potential vorticity, respectively, while $F$ is the forcing function; $h_{1}=0.3$ and $h_{2}=0.7$ are nondimensional thicknesses of the lower and upper atmospheric layers, $R_{d}=383 \mathrm{~km}$ is the Rossby radius of deformation, $f_{0}=$ $10^{-4} \mathrm{~s}^{-1}$ is the Coriolis parameter, $\beta=2 \times 10^{-11} \mathrm{~m}^{-1}$ $\mathrm{s}^{-1}$ is the gradient of the Coriolis parameter at $45^{\circ} \mathrm{N}, k$ is the bottom drag, which will be used as a control parameter, $A_{H}=-1.5 \times 10^{16} \mathrm{~m}^{4} \mathrm{~s}^{-1}$ is the superviscosity coefficient, and $J(A, B) \equiv(\partial A / \partial x)(\partial B / \partial y)-(\partial A /$ $\partial y)(\partial B / \partial x)$ is the Jacobian. Additional damping terms with the characteristic time scales of $\left[k^{(1)}\right]^{-1}=23$ days, $\left[k^{(2)}\right]^{-1}=29$ days, and $\left[k^{(3)}\right]^{-1}=37$ days are included, following Vautard et al. (1988).

The net, incident less reflected, short-wave radiation at the top of the atmosphere $R$, expressed in $\mathrm{W} \mathrm{m}^{-2}$, is

$$
R=190-165 \sin \left(2 y / a_{E}\right),
$$

where $a_{E}=6400 \mathrm{~km}$ is the radius of the earth. We parameterize other heat fluxes through the sea surface temperature $T_{\mathrm{s}}$ and the atmospheric temperature $T_{a}$; the latter is proportional to the baroclinic streamfunction $\tau$, as in Kravtsov et al. (2003).

Over the ocean, the atmospheric forcing function is

$$
F=\frac{1}{\rho_{a} c_{P, a} \Delta \theta_{s}}\left(O+H_{\mathrm{SL}}-2 B\right),
$$

where $\Delta \theta_{\mathrm{s}}=50 \mathrm{~K}$ is the difference in potential temperature between the layers, $\rho_{a}=1.27 \mathrm{~kg} \mathrm{~m}^{-3}$ is the representative atmospheric density, and $c_{P, a}=1000 \mathrm{~J}$ $\mathrm{kg}^{-1} \mathrm{~K}^{-1}$ is the atmospheric heat capacity. Neglecting the heat capacity and conductivity of the land surface results in the forcing function

$$
F=\frac{1}{\rho_{a} c_{P, a} \Delta \theta_{s}}(R-B),
$$

valid over land.

The atmospheric back radiation $B$ and the outgoing oceanic longwave radiation absorbed by the atmosphere $O$ are linear functions of $T_{a}$ and sea surface temperature $T_{s}$, respectively (Kravtsov et al. 2003). The heat exchange between the ocean and the atmosphere is parameterized using a standard bulk formula (Gill 1982):

$$
H_{\mathrm{SL}}=\rho_{a} U\left\{C_{h} c_{\mathrm{P}, \mathrm{a}}\left(T_{s}-T_{a}\right)+C_{e} L\left[q\left(T_{s}\right)-q\left(T_{a}\right)\right]\right\} .
$$

Here, $L=2.5 \times 10^{6} \mathrm{~J} \mathrm{~kg}^{-1}$ is the latent heat of vaporization of water, $C_{h}=0.001$ and $C_{e}=0.0015$. The sea level wind $U$ is computed through the wind stress $\tau^{(x)}$, $\tau^{(y)}$ as

$$
U=\left[\left(\tau^{(x) 2}+\tau^{(y) 2}\right)^{(1 / 2)} /\left(\rho_{a} C_{d}\right)\right]^{(1 / 2)},
$$

where $\tau^{(x)}, \tau^{(y)}$ are found, in turn, through the atmospheric lower-layer velocities $u, v$ as

$$
\left[\tau^{(x)}, \tau^{(y)}\right]=\rho_{a} H_{a} k(u-v, u+v)
$$

and $C_{d}=0.0012$. The specific humidity $q(T)$ is determined via the linearized Clapeyron-Clausius equation

$$
q(T)=3.77 \times 10^{-3}(1+0.07 T) .
$$

Finally, sea surface temperature in our atmosphereonly model is specified as

$$
T_{s}=13-17 \sin \left(2 y / a_{E}\right) .
$$

\section{APPENDIX B}

\section{Transition to Chaos}

We consider here in greater detail the behavior of the model for very high bottom drag, before transition to bimodality occurs (see Fig. 3a). The interest in this range of parameters is twofold: (i) it is the only range where active synoptic eddy feedback occurs; and (ii) it includes the type of global bifurcation that has attracted considerable attention recently in both the atmospheric and oceanic literature.

For $k^{-1}=0.39$ day, the model has a single stable equilibrium, as described in section 3. As the bottom drag decreases, the model's behavior becomes increasingly more complex. In Fig. B1, we plot the time series of the leading PC of the standard combined, barotropic and baroclinic streamfunction field for $k^{-1}>0.39$ day. For $k^{-1}=0.46$ day (Fig. B1a), the model exhibits a highly nonlinear but still perfectly periodic relaxation oscillation, with a period of about 740 days. The strong spikes are associated with abrupt drops in jet velocity and alternate with long time intervals during which the system stays close to the unstable steady state that resembles the stable equilibrium obtained for $k^{-1}=0.39$ day (light solid line in Fig. 3). As the bottom drag decreases further, the oscillation becomes more and more irregular, while its mean frequency increases; concomitantly, the oscillation's negative and positive phases become increasingly more similar in duration and amplitude (Figs. B1b-e).

For $k^{-1}=1.16$ days, the model trajectory loses regularity completely and becomes chaotic (Fig. B1f). The leading PC is now associated with the shifts of the jet, rather than with changes in the jet intensity, as in Figs. B1a-e. The trajectory jumps between two regimes: the low-latitude regime in this case is maintained by the eddies as described in section 5, while the high-latitude regime is associated with the presence of the unstable high-latitude equilibrium. As the bottom drag decreases even further, the instability of the high-latitude equilibrium increases, while the eddy-driven lowlatitude state migrates toward the center of the channel (Fig. 3a). Therefore, the probability for the system to 

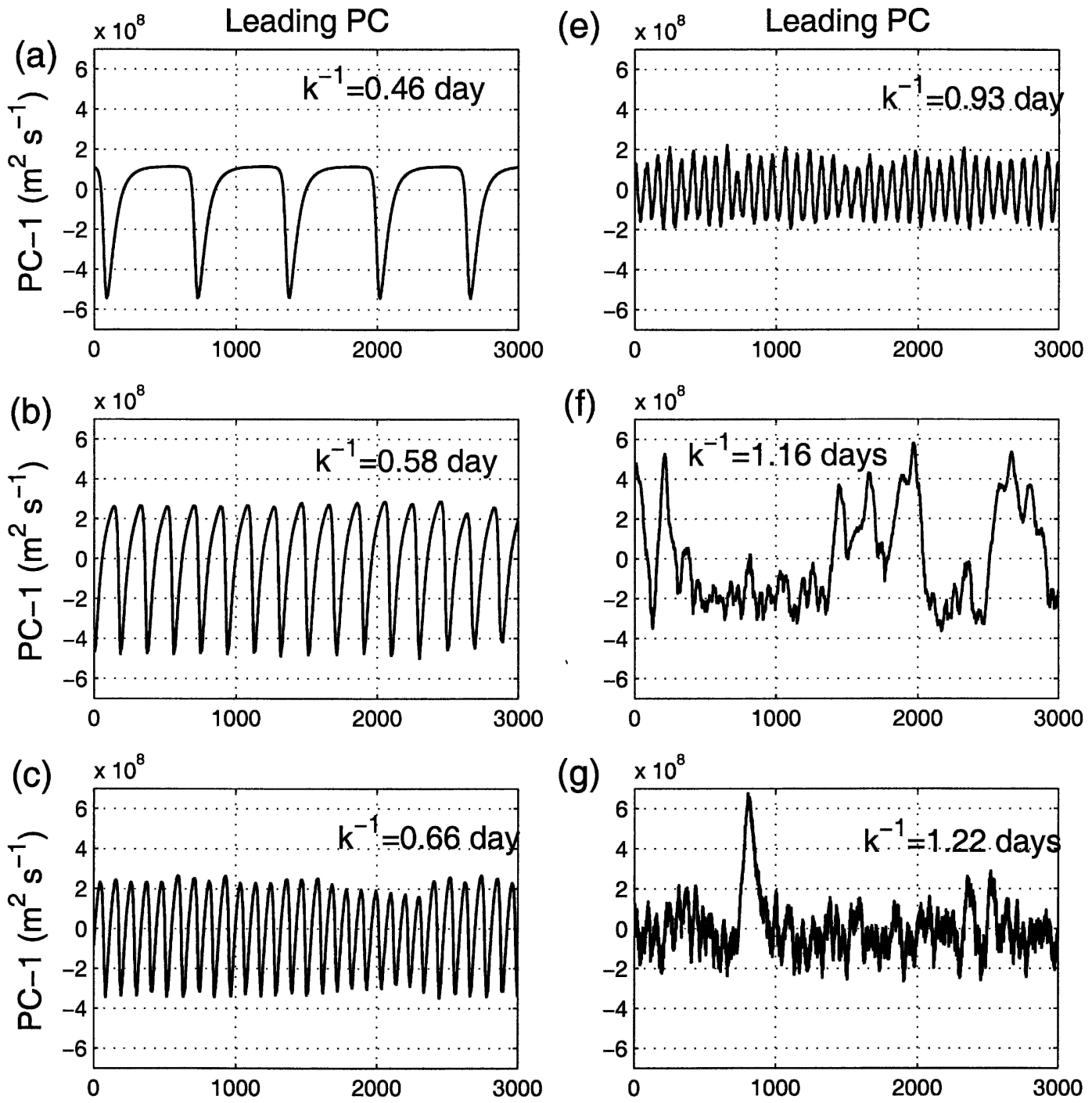

(g)
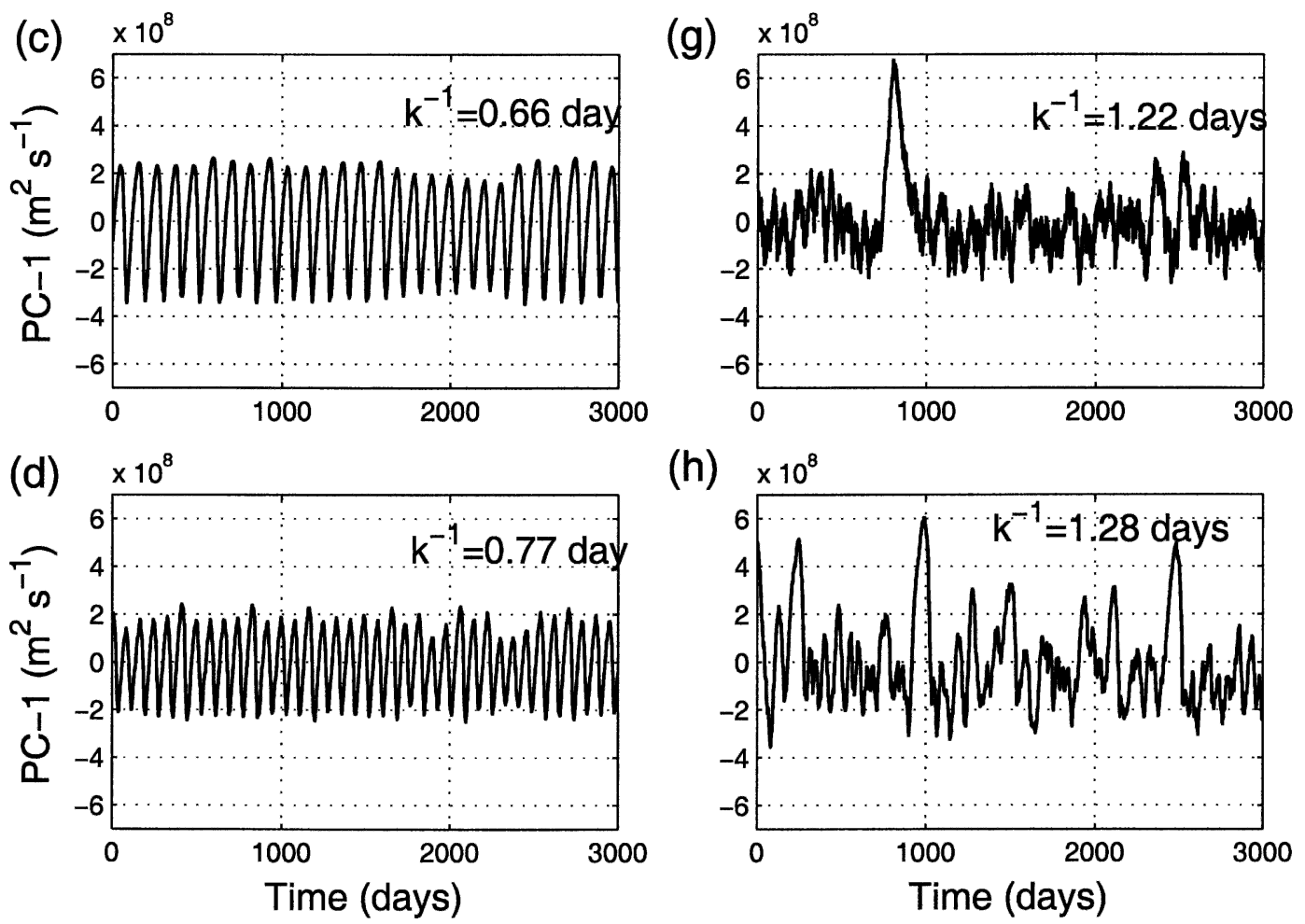

(h)

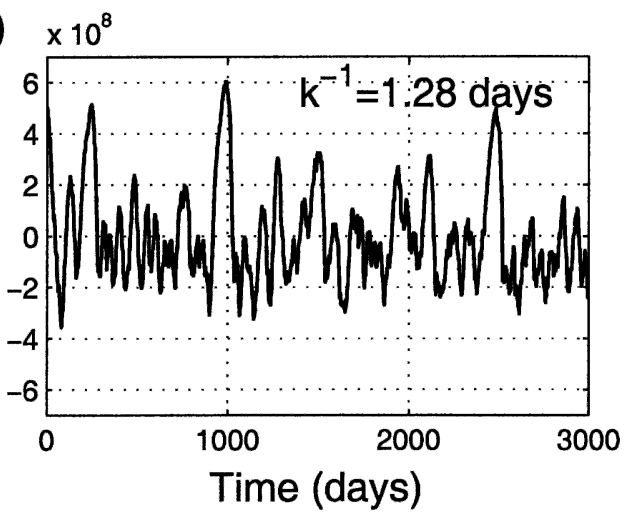

FIG. B1. Transition to chaos: time series of the leading PC of the combined barotropic and baroclinic streamfunction field $\left(\mathrm{m}^{2} \mathrm{~s}^{-1}\right)$ for values of $k^{-1}$ between 0.46 and 1.28 days, as listed in each panel.

spend significant time intervals near the unstable equilibrium decreases and its variability is characterized now by irregular shifts of the jet about the eddymaintained state (Figs. B1g-h). This behavior persists for all lower values of the bottom drag and has been described in detail in sections 3, 4, and 5 .

The baroclinic wave- 5 dynamics plays a crucial role in the relaxation oscillation of Fig. B1a; this wave ap- 


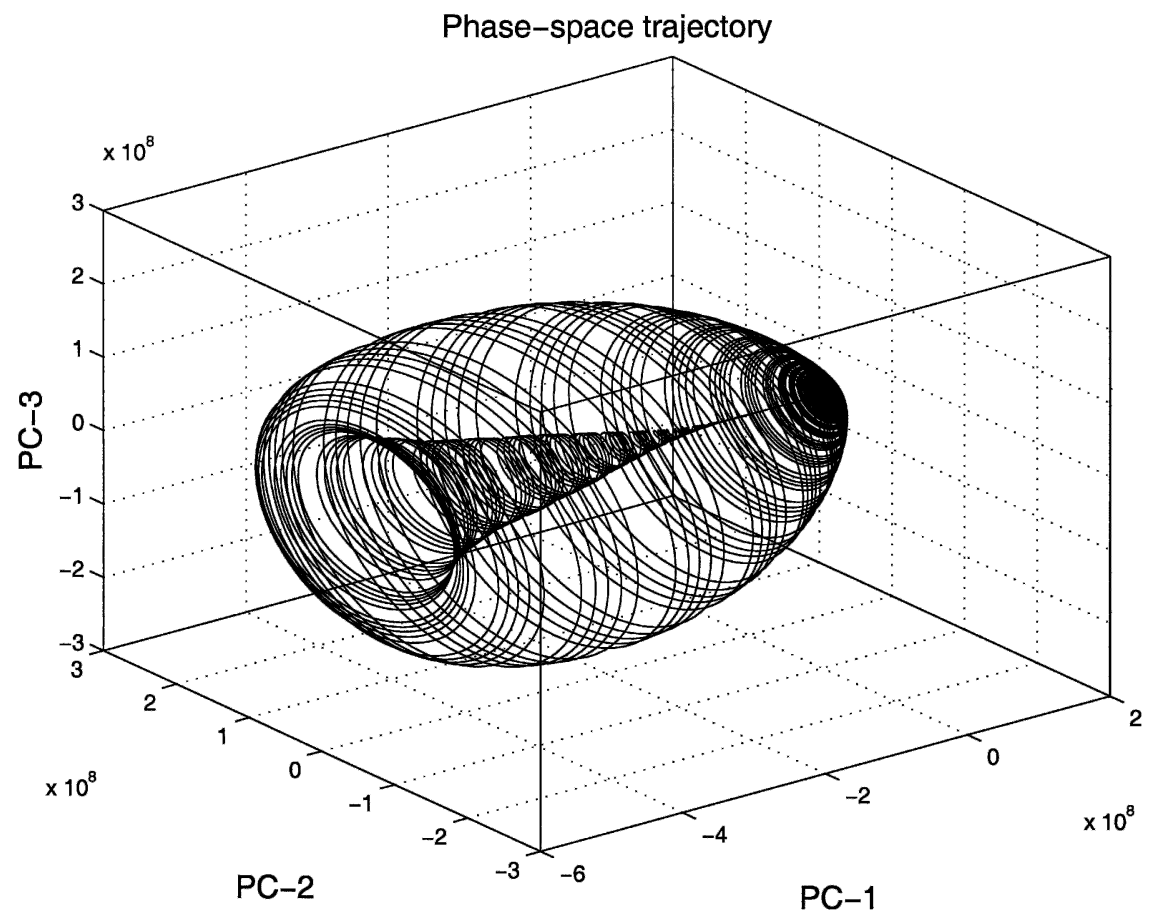

FIG. B2. Model trajectory in the subspace spanned by the three leading EOFs of the combined barotropic and baroclinic streamfunction field, for $k^{-1}=0.46$ day; compare with PC-1 time series of Fig. B1a. The time interval plotted here equals 3000 days, as in Figs. B1a-h.

pears as the pair PC 2-PC 3 in the EOF decomposition (not shown). The model trajectory in the phase subspace of the leading three PCs is plotted in Fig. B2 for $k^{-1}=0.46$ day; EOFs $1-3$ account for $98 \%$ of the total variance at this value of $k^{-1}$.

The unstable equilibrium is at the apex of the bowlshaped object in Fig. B2. Near this apex, wave 5 slowly decays, as it intensifies the zonal-mean jet and increases its baroclinic instability. As a result, the trajectory is ejected along the unstable direction of the apex (the axis of symmetry of the bowl), giving rise to a spike event like those shown in Fig. 14a. During this event, wave 5 first gains energy rapidly, as the trajectory fans out to the lip of the bowl, and then loses energy more slowly by nonlinear damping. The trajectory thus spirals in again on the apex, following its stable directions, and the cycle repeats.

The dynamics of the oscillations shown in Figs. B1b-e is similar. A transition to the chaotic behavior in Fig. B1f is accompanied by the change in the wavenumber of the dominant baroclinic wave from 5 to 6 ; wave 5 recovers its dominance over wave 6 around $k^{-1}=2$ days (see Fig. 9b).

Figure B2 provides a striking illustration of a homoclinic orbit in a high-dimensional system; recall that the number of discrete variables equals $128 \times 41 \times 2=$ 10 496. It is possible that the orbit in the full phase space misses exact return to the unstable fixed point at the apex of the bowl but, if so, the exact homoclinic orbit must still be very close in phase-parameter space.
Both homoclinic and heteroclinic orbits give rise to so-called global bifurcations (Guckenheimer and Holmes 2002). A homoclinic orbit is a closed orbit, like the one in Fig. 15, that reconnects an unstable equilibrium to itself and arises as the unstable manifold of this equilibrium, that is the nonlinear deformation of its unstable subspace, merges smoothly with its stable manifold. Heteroclinic orbits connect two or more unstable equibria, as the unstable manifold of one such equilibrium merges smoothly with the stable equilibrium of another one.

Global bifurcations are thus genuinely nonlinear phenomena that involve possibly large portions of a system's phase space and not merely the nonlinear saturation of an essentially linear instability. Among the simpler, local bifurcations, saddle-node and pitchfork bifurcations arise due to the nonlinear saturation of a purely exponential instability, while Hopf bifurcations arise because of the nonlinear saturation of an oscillatory instability. These local bifurcations, well known by now in the atmospheric and oceanic literature, are very robust and thus relatively easy to compute, while global bifurcations are much more sensitive and much harder to compute, even in fairly low-dimensional systems.

In the atmospheric literature, Lorenz (1963) was the first to mention the potential role of a heteroclinic orbit in transition to chaos for a low-order model with 14 variables; he already connected this potential role to the mechanics of vacillation, in a rotating annulus and the NH circulation. Ghil and Childress (1987, sections 
5.3 and 5.5) discussed at length this role, mentioning more specifically the Shilnikov (1965) mechanism of transition to chaos in the same context. They also conjectured that homoclinic orbits might play an important role in flow regime persistence (see discussion of Fig. 6.12 in section 6.4 of Ghil and Childress 1987).

Kimoto and Ghil (1993) and Weeks et al. (1997) mentioned the possible role of heteroclinic connections as giving rise to transitions between regimes, in $\mathrm{NH}$ observations and a rotating annulus with topography, respectively. Kondrashov et al. (2004) also discussed this role more recently for transitions between the realistic NH regimes of the Marshall and Molteni (1993) three-layer QG model. Hetero- and homoclinic orbits were shown to play an important role in the interannual and interdecadal LFV of the midlatitude ocean's winddriven circulation (Meacham 2000; Chang et al. 2001; Nadiga and Luce 2001; Simonnet et al. 2003a,b).

Crommelin $(2002,2003)$ explored in detail the role of such orbits in a low-order and an intermediate-order model of the extratropical atmosphere, respectively; the former had 10, the latter 231 variables. By comparison with this earlier work, our results in Fig. B2 here refer to a model with over $10^{4}$ variables and are exact; they also relate the homoclinic orbit to a wellunderstood physical mechanism, namely the interaction between a baroclinic wave and the mean flow.

\section{REFERENCES}

Akahori, K., and S. Yoden, 1997: Zonal flow vacillation and bimodality of baroclinic eddy life cycles in a simple global circulation model. J. Atmos. Sci., 54, 2349-2361.

Branstator, G. W., 1992: The maintenance of low-frequency atmospheric anomalies. J. Atmos. Sci., 49, 1924-1945.

- 1995: Organization of storm track anomalies by recurring low-frequency circulation anomalies. J. Atmos. Sci., 52, 207226.

— vorticity equation. J. Atmos. Sci., 46, 1799-1814.

Brunet, G., 1994: Empirical normal-mode analysis of atmospheric data. J. Atmos. Sci., 51, 932-952.

Cai, M., and H. M. Van den Dool, 1994: Dynamical decomposition of low- and high-frequency tendencies. J. Atmos. Sci., 51, 2086-2100.

Chang, K.-I., M. Ghil, K. Ide, and C.-C. A. Lai, 2001: Transition to aperiodic variability in a wind-driven double-gyre circulation model. J. Phys. Oceanogr., 31, 1260-1286.

Charney, J. G., and J. G. DeVore, 1979: Multiple flow equilibria in the atmosphere and blocking. J. Atmos. Sci., 36, 1205-1216.

Corti, S., A. Giannini, S. Tibaldi, and F. Molteni, 1997: Patterns of low-frequency variability in a three-level quasi-geostrophic model. Climate Dyn., 13, 883-904.

Crommelin, D. T., 2002: Homoclinic dynamics: A scenario for atmospheric ultra-low-frequency variability. J. Atmos. Sci., 59, 1533-1549.

_- 2003: Regime transitions and heteroclinic connections in a barotropic atmosphere. J. Atmos. Sci., 60, 229-246.

Da Costa, E., and R. Vautard, 1997: A qualitative realistic loworder model of the extratropical low-frequency variability built from long records of potential vorticity. J. Atmos. Sci., 54, 1064-1084.

Deser, C., 2000: On the teleconnectivity of the "Arctic Oscillation." Geophys. Res. Lett., 27, 779-782.
Farrell, B. F., and P. J. Ioannou, 1993: Stochastic forcing of the linearized Navier-Stokes equations. Phys. Fluids A, 5, 26002609.

— and _ 1995: Stochastic dynamics of the midlatitude atmospheric jet. J. Atmos. Sci., 52, 1642-1656.

Feldstein, S. B., 2000: Is interannual zonal mean flow variability simply climate noise? J. Climate, 13, 2356-2362.

- and S. Lee, 1996: Mechanisms of zonal index variability in an aquaplanet GCM. J. Atmos. Sci., 53, 3541-3555.

—, and - 1998: Is the atmospheric zonal index driven by an eddy feedback? J. Atmos. Sci., 55, 3077-3086.

Gardiner, C., 1983: Handbook of Stochastic Methods. SpringerVerlag, $442 \mathrm{pp}$.

Ghil, M., and S. Childress, 1987: Topics in Geophysical Fluid Dynamics: Atmospheric Dynamics, Dynamo Theory and Climate Dynamics. Springer-Verlag, 485 pp.

- , and A. W. Robertson, 2000: Solving problems with GCMs: General circulation models and their role in the climate modeling hierarchy. General Circulation Model Development: Past, Present and Future, D. Randall, Ed., Academic Press, 285-325.

— phase space: A pathway to long-range forecasting? Proc. Natl. Acad. Sci., 99 (Suppl. 1), 2493-2500.

_ , Y. Feliks, and L. U. Sushama, 2002: Baroclinic and barotropic aspects of the wind-driven ocean circulation. Physica D, 167, 1-35.

Gill, A., 1982: Atmosphere-Ocean Dynamics. Academic Press, $662 \mathrm{pp}$.

Greatbatch, R. J., 1987: A model for the inertial recirculation of a gyre. J. Mar. Res., 45, 601-634.

—, 1988: On the scaling of the inertial subgyres. Dyn. Atmos. Oceans, 11, 265-285.

Guckenheimer, J., and P. Holmes, 2002: Nonlinear Oscillations, Dynamical Systems, and Bifurcations of Vector Fields. 2d ed. Springer-Verlag, $475 \mathrm{pp}$.

Hartmann, D. L., 1995: A PV view of zonal flow vacillation. $J$. Atmos. Sci., 52, 2561-2576.

_ and F. Lo, 1998: Wave-driven zonal flow vacillation in the Southern Hemisphere. J. Atmos. Sci., 55, 1303-1315.

Hurrel, J. E., 1995: Decadal trends in the North Atlantic Oscillation: Regional temperatures and precipitation. Science, 269, 676-679.

Illari, L., 1984: A diagnostic study of the potential vorticity in a warm blocking anticyclone. J. Atmos. Sci., 41, 3518-3526.

Itoh, H., and M. Kimoto, 1999: Weather regimes, low-frequency oscillations, and principal patterns of variability: A perspective of extratropical low-frequency variability. J. Atmos. Sci., 56, 2684-2705.

James, I. N., and L. J. Gray, 1986: Concerning the effect of surface drag on the circulation of a baroclinic planetary atmosphere. Quart. J. Roy. Meteor. Soc., 112, 1231-1250.

Kidson, J. W., and I. G. Watterson, 1999: The structure and predictability of the "high-latitude mode" in the CSIRO9 general circulation model. J. Atmos. Sci., 56, 3859-3873.

Kimoto, M., and M. Ghil, 1993: Multiple flow regimes in the Northern Hemisphere winter. Part II: Sectorial regimes and preferred transitions. J. Atmos. Sci., 50, 2645-2673.

_, F.-F. Jin, M. Watanabe, and N. Yasutomi, 2001: Zonal-eddy coupling and a neutral mode theory for the Arctic Oscillation. Geophys. Res. Lett., 28, 737-740.

Kondrashov, D., K. Ide, and M. Ghil, 2004: Weather regimes and preferred transition paths in a three-level quasigeostrophic model. J. Atmos. Sci., 61, 568-587.

Koo, S., 2002: Nonlinear aspects of atmospheric zonal-flow vacillation. Ph.D. thesis, University of California, Los Angeles, $163 \mathrm{pp}$. 
— of atmospheric zonal-flow vacillation. Chaos, 12, 300-309.

-, A. W. Robertson, and M. Ghil, 2003: Multiple regimes and low-frequency oscillations in the Southern Hemisphere's zonal-mean flow. J. Geophys. Res., 107, 4596, doi:10.1029/ 2001JD001353.

Kravtsov, S., and A. W. Robertson, 2002: Midlatitude oceanatmosphere interaction in an idealized coupled model. Climate Dyn., 19, 693-711.

- - - and M. Ghil, 2003: Low-frequency variability in a baroclinic $\beta$ channel with land-sea contrast. J. Atmos. Sci., 60, 2267-2293.

Lee, S., and S. B. Feldstein, 1996: Mechanisms of zonal index evolution in a two-layer model. J. Atmos. Sci., 53, 2232-2246.

Legras, B., and M. Ghil, 1985: Persistent anomalies, blocking and variations in atmospheric predictability. J. Atmos. Sci., 42, $433-471$.

Lorenz, D. J., and D. L. Hartmann, 2001: Eddy-zonal flow feedback in the Southern Hemisphere. J. Atmos. Sci., 58, 33123327.

— , and - 2003: Eddy-zonal flow feedback in the Northern Hemisphere winter. J. Climate, 16, 1212-1227.

Lorenz, E. N., 1963: The mechanics of vacillation. J. Atmos. Sci., 20, 448-464.

Marshall, J., and F. Molteni, 1993: Toward a dynamical understanding of atmospheric weather regimes. J. Atmos. Sci., 50, 1792-1818.

— waves. J. Atmos. Sci., 47, 963-978.

McWilliams, J. C., 1977: A note on a consistent quasi-geostrophic model in a multiply connected domain. Dyn. Atmos. Oceans, 1, 427-441.

Meacham, S. P., 2000: Low-frequency variability in the winddriven circulation. J. Phys. Oceanogr., 30, 269-293.

Metz, W., 1994: Singular modes and low-frequency atmospheric variability. J. Atmos. Sci., 51, 1740-1753.

Nadiga, B. T., and B. P. Luce, 2001: Global bifurcation of Shilnikov type in a double-gyre ocean model. J. Phys. Oceanogr., 31, 2669-2690.

Namias, J., 1953: Thirty-Day Forecasting: A Review of a TenYear Experiment. Meteor. Monogr., No. 6, Amer. Meteor. Soc., 83 pp.

North, G. R., T. L. Bell, R. F. Cahalan, and F. J. Moeng, 1982: Sampling errors in the estimation of empirical orthogonal functions. Mon. Wea. Rev., 110, 699-706.

Oppenheim, A. V., and R. W. Schafer, 1989: Discrete-Time Signal Processing. Prentice Hall, 879 pp.

Pedlosky, J., 1981: Resonant topographic waves in barotropic and baroclinic flows. J. Atmos. Sci., 38, 2626-2641.

— 1987: Geophysical Fluid Dynamics. 2d ed. Springer-Verlag, $621 \mathrm{pp}$.

Preisendorfer, R. W., 1988: Principal Component Analysis in Meteorology and Oceanography. Elsevier, $425 \mathrm{pp}$.

Reinhold, B. B., and R. T. Pierrehumbert, 1982: Dynamics of weather regimes: Quasi-stationary waves and blocking. Mon. Wea. Rev., 110, 1105-1145.

Robertson, A. W., 2001: Influence of ocean-atmosphere interaction on the Arctic Oscillation in two general circulation models. J. Climate, 14, 3240-3254.

, and W. Metz, 1989: Three-dimensional instability of persistent anomalous large-scale flows. J. Atmos. Sci., 46, 2783 2801.
—- and W. Metz, 1990: Transient eddy feedbacks derived from linear theory and observations. J. Atmos. Sci., 47, 2743-2764.

Robinson, W., 1991: The dynamics of low-frequency variability in a simple model of the global atmosphere. J. Atmos. Sci., 48, 429-441.

_ 1996: Does eddy feedback sustain variability in the zonal index? J. Atmos. Sci., 53, 3556-3569.

_ 2000: A baroclinic mechanism for the eddy feedback on the zonal index. J. Atmos. Sci., 57, 415-422.

Schuss, Z., 1980: Theory and Applications of Stochastic Differential Equations. Wiley, 321 pp.

Shilnikov, L. P., 1965: A case of the existence of a denumerable set of periodic motions. Sov. Math. Dokl., 6, 163-166.

Shutts, G. J., 1983: The propagation of eddies in diffluent jet streams: Eddy vorticity forcing of blocking flow fields. Quart. J. Roy. Meteor. Soc., 109, 737-761.

Simonnet, E., M. Ghil, K. Ide, R. Temam, and S. Wang, 2003a: Low-frequency variability in shallow-water models of the wind-driven ocean circulation. Part I: Steady-state solutions. J. Phys. Oceanogr., 33, 712-728.

,,,,---- and,- 2003 b: Low-frequency variability in shallow-water models of the wind-driven ocean circulation. Part II: Time-dependent solutions. J. Phys. Oceanogr., 33, 729-752.

Swanson, K. L., 2000: Stationary wave accumulation and generation of low-frequency variability on zonally varying flows. $J$. Atmos. Sci., 57, 2262-2280.

Thompson, D. W. J., and J. M. Wallace, 2000: Annular modes in the extratropical circulation. Part I: Month-to-month variability. J. Climate, 13, 1000-1016.

,-- and G. C. Hegerl, 2000: Annular modes in the extratropical circulation. Part II: Trends. J. Climate, 13, 1018-1036.

Thorncroft, C. D., B. J. Hoskins, and M. E. McIntyre, 1993: Two paradigms of baroclinic-wave life-cycle behavior. Quart. J. Roy. Meteor. Soc., 119, 17-55.

Vautard, R., B. Legras, and M. Deque, 1988: On the source of midlatitude low-frequency variability. Part I: A statistical approach to persistence. J. Atmos. Sci., 45, 2811-2843.

_, P. Yiou, and M. Ghil, 1992: Singular-spectrum analysis: A toolkit for short, noisy chaotic signals. Physica D, 58, 95-126.

Wallace, J. M., 1983: The climatological mean stationary waves: Observational evidence. Large-Scale Dynamical Processes in the Atmosphere. B. J. Hoskins and R. P. Pearce, Eds., Academic Press, 27-53.

_ 2 2000: North Atlantic Oscillation/annular mode: Two paradigms-One phenomenon. Quart. J. Roy. Meteor. Soc., 126, 791-805.

Watanabe, M., and F.-F. Jin, 2003: A moist baroclinic model: Coupled dynamical-convective response to El Niño. J. Climate, 16, 1121-1139.

Weeks, E. R., Y. Tian, J. S. Urbach, K. Ide, H. L. Swinney, and M. Ghil, 1997: Transitions between blocked and zonal flows in a rotating annulus with topography. Science, 278, 1598-1601.

Weisheimer, A., M. V. Kurgansky, K. Dethloff, and D. R. Handorf, 2003: Extratropical low-frequency variability in a threelevel quasi-geostrophic atmospheric model with different spectral resolution. J. Geophys. Res., 108, 4171, doi:10.1029/ 2001JD001282.

Yu, J.-Y., and D. L. Hartmann, 1993: Zonal flow vacillation and eddy forcing in a simple GCM of the atmosphere. J. Atmos. Sci., 50, 3244-3259. 